\title{
Towards pore network modelling of spontaneous imbibition: contact angle dependent invasion patterns and the occurrence of dynamic capillary barriers
}

\author{
Saideep Pavuluri ${ }^{1}$ (D) Julien Maes ${ }^{1} \cdot$ Jianhui Yang $^{2} \cdot$ Mohamed Regaieg $^{2} \cdot$ Arthur Moncorgé $^{2} \cdot$ Florian Doster $^{1}$
}

Received: 24 October 2018 / Accepted: 31 May 2019/ Published online: 1 July 2019

(C) The Author(s) 2019

\begin{abstract}
Imbibition is an important process encountered in many porous media applications. At the pore scale, pore network models (PNM) are computationally efficient and can model drainage accurately. However, using PNM to model imbibition still remains a challenge due to the complexities encountered in understanding pore-scale flow phenomena related to pore body filling (PBF) and snap-off along with the relative competition between these events. In this work, we use direct numerical simulations (DNS) to revisit the basic principles of PBF in a two-dimensional synthetic pore geometry. We notice that PBF during spontaneous imbibition is dependent on several parameters such as shape of the transition zone, contact angle and the fluid properties like density. The interactions between these parameters are investigated in a quantitative manner. We demonstrate the existence of a critical contact angle $\theta_{c}$ and a barrier contact angle $\theta_{b} . \theta_{c}$ depends on the shape of the pore geometry, whereas $\theta_{b}$ depends on the pore geometry, contact angle and fluid properties. For a system comprising of light fluids, $\theta_{b}$ is only slightly larger than $\theta_{c}$; whereas for a system occupied by dense fluids, $\theta_{b}$ is notably larger than $\theta_{c}$. The contact angle of the wetting phase $\theta$ in relation to $\theta_{c}$ and $\theta_{b}$ decides if the wetting phase can imbibe a pore body. Imbibition always occurs if $\theta<\theta_{c}$. For $\theta>\theta_{c}$, we observe capillary barrier zones in which capillary forces accompany viscous forces to resist spontaneous imbibition. For this case, we observe smooth transition of the meniscus curvature while the meniscus enters and exits capillary barrier zones. For $\theta_{c} \leq \theta \leq \theta_{b}$, inertia assists the wetting phase to overcome resisting forces and imbibe the pore space. For $\theta>\theta_{b}$, the resisting forces dominate over inertia so that the wetting phase cannot imbibe the pore space. For the synthetic pore geometries investigated, we provide analytical and semi-analytical expressions to determine $\theta_{c}$ and the position of capillary barrier zones respectively. The barrier contact angle $\theta_{b}$ is computed numerically for several inertial systems and for various shapes of the synthetic pore geometry. The results of this quantitative analysis can be utilised to improve the existing pore filling rules and predictive capabilities of PNM used for two-phase flows.
\end{abstract}

Keywords Pore body filling · Spontaneous imbibition · Dynamic capillary barriers · Pore geometry $\cdot$ Contact angle . Inertia

\section{Introduction}

Multiphase flows occur in a wide range of industrial and engineering applications such as in the recovery of hydrocarbons, carbon capture and sequestration, fuel cells and

\section{Electronic supplementary material The online version of} this article (https://doi.org/10.1007/s10596-019-09842-7) contains supplementary material, which is available to authorised users.

Saideep Pavuluri

saideep.pavuluri@pet.hw.ac.uk

Extended author information available on the last page of the article. lab-on-a-chip devices. The Darcy scale flow behaviour is a cumulative collection of the flow physics occurring within each of the millions of interconnected pores. Despite several decades of research from the pioneering works of $[13,14]$, many flow phenomena that occur at the pore scale still lack profound understanding. The pore geometry along with the fluid properties and contact angle govern the magnitude of inertial, viscous and capillary forces acting within the system $[8,16]$ which in turn govern the displacement pattern of the fluids. In recent times, advancements in imaging the pore spaces of core plugs [23] and micromodels [26] have resulted in capturing interesting flow phenomena such as viscous and capillary fingering, snap-off and cooperative pore body filling. However, 
numerical studies are indispensable for a quantitative understanding of the dynamic flow processes and for parameter optimisations.

Pore network models (PNM) and direct numerical simulations (DNS) are used to investigate fluid displacements at the pore scale. DNS are based on the first principles of physics and provides a complete insight regarding the pore scale flow dynamics. However, DNS are computationally expensive (for reference: flow analysis on a micro-CT takes a week). On the other hand, PNM are computationally efficient (flow analysis on a micro-CT takes less than an hour) but rely on invasion-percolation rules and on additional flow assumptions thereby sacrificing accuracy. PNM are efficient in modelling drainage, where a non-wetting phase invades to displace the wetting phase in a piston-type motion [13]. The nature of fluid displacement is governed by the viscosity ratio and capillary number resulting in fingering (viscous or capillary) or stable displacement flow patterns [24]. During imbibition, where a wetting phase invades to displace the non-wetting phase, complex displacement flow patterns are observed [14, 23]: (1) piston-type motion, (2) pore body filling (cooperative when the pore body is connected to multiple throats) and (3) snap-off. Further, the competition between piston-type motion, pore body filling (PBF) and snap-off results in either filling of the pores by the wetting phase thereby recovering the non-wetting phase or in trapping of the non-wetting phase in the pore bodies.

Imbibition is a common displacement process encountered during hydrocarbon recovery as majority of minerals in hydrocarbon reservoirs show affinity towards water prior to ageing (long-term exposure to oil) [9, 27], making the surface of the pore initially water-wet. Even after primary drainage, the reservoir turns to a mixed-wet state as the larger pores most probably come in contact with hydrocarbons. These larger pores can then be subjected to wettability alterations, whereas the smaller pores continue being occupied by the wetting phase and remain water-wet [22]. In recent times, imbibition has gathered more attention and has been studied experimentally $[23,26]$ and numerically $[8,20]$ giving insights regarding the inter-pore and intrapore fluid displacement processes. Focussing exclusively on PBF, Ferrari and Lunati [8] have shown the occurrence of meniscus reconfigurations at the corners of a square and circular pore during forced imbibition and used the principles of energy balance to study meniscus reconfigurations analytically. Further, they also notice that the meniscus reconfigurations have their own time scales and velocities. Zacharoudiou et al. [25] have experimentally noticed transition in meniscus curvature (from imbibition curvature to drainage curvature) during quasi-static imbibition in a sharp throat-pore body system. Further, using numerical investigations, they show that these meniscus oscillations are not only limited to forced imbibition scenarios but also occur for spontaneous imbibition flows in a sharp throat-pore body system.

We revisit the concept of PBF in this paper by investigating meniscus dynamics that occur at the transition zone (pore space that connects a pore throat and pore body) having different shapes. PNM for PBF currently use rules that capture the qualitative behaviour of displacement patterns [4]. For our investigation, we consider a synthetic two-dimensional pore geometry and spontaneous imbibition flow conditions. We uncover the concept of capillary barriers which are seen to play a significant role in choosing the invasion paths of the wetting phase during spontaneous imbibition. The occurrence of capillary barrier zones are linked to the shape of transition zone and contact angle of the fluid. However, PNM do not take into account the transition zone shape while modelling imbibition. We quantitatively analyse PBF using DNS for different scenarios by tuning the following dependent parameters: pore geometry (transition zone shape, aspect ratio), contact angle and the fluid properties. To showcase the importance of acknowledging capillary barriers, we investigate spontaneous imbibition at different contact angles in a pore doublet comprising of two pores having different size and transition zone shape. Although the focus of this manuscript is on contact angle dependent invasion patterns, this work also provides an insight regarding the optimal design of porous membranes to avoid or achieve spontaneous imbibition which is vital for the manufacture of fuel cell membranes and during inkjet printing.

The manuscript is organised as follows: Section 2 introduces the numerical formulation used for the study along with the contact angle and partial slip wall boundary conditions. Section 3 discusses the shape of the two-dimensional synthetic pore geometry considered for investigation along with the analytic and semi-analytic expressions to determine the critical contact angle and locate the position of the capillary barrier zones respectively. The numerical setup is discussed in Section 4 and the results for various cases are discussed in Section 5. Finally, we end the paper with concluding remarks.

\section{Equations governing two-phase flow}

The isothermal dynamics of two immiscible and incompressible fluids are governed by the Navier-Stokes Equations (NSEs) for each fluid. The NSEs for individual fluids are coupled through a series of boundary conditions at the interface [2]. Hence, using this approach requires solving a challenging moving boundary problem.

The volume-of-fluid (VOF) method simplifies the moving boundary problem by treating the two-phase fluid system as a single mixture [10]. VOF uses a bounded colour 
function $\alpha=[0,1]$ to distinguish the fluids. The colour function represents the normalised volume of a specific phase in the control volumes.

The NSEs for the VOF mixture comprise of the mass conservation,

$\nabla \cdot \mathbf{U}=0$

and the momentum conservation equations,

$\frac{\partial(\rho \mathbf{U})}{\partial t}+\nabla \cdot(\rho \mathbf{U U})=-\nabla p+\nabla \cdot \mu\left(\nabla \mathbf{U}+\nabla \mathbf{U}^{T}\right)+\mathbf{F}_{\mathrm{b}}+\mathbf{F}_{\mathrm{cap}}$,

where $\mathbf{U}$ denotes the velocity field, $\rho$ the density, $t$ the time, $p$ the pressure, $\mu$ the dynamic viscosity, $\mathbf{F}_{\mathrm{b}}$ the external body forces and $\mathbf{F}_{\text {cap }}$ the capillary forces. The density $\rho$ and dynamic viscosity $\mu$ of the mixture are given by,

$\rho=\rho_{w} \alpha+\rho_{n}(1-\alpha)$,

$\mu=\mu_{w} \alpha+\mu_{n}(1-\alpha)$,

where the indices $w$ and $n$ denote the two fluids in the system. The capillary forces are given by,

$\mathbf{F}_{\text {cap }}=\sigma k \mathbf{n}_{\mathrm{I}} \delta_{\mathrm{I}}$

where $\sigma$ is the surface tension between fluids, $k$ is the interface curvature, $\mathbf{n}_{\mathrm{I}}$ is the unit interface normal and $\delta_{\mathrm{I}}$ is a Dirac function representing a sharp interfacial region where the capillary forces act. In this manuscript, we refer to the interface between two fluids that is in contact with the wall as a meniscus. The terms such as interface curvature and interface normal refer to the mentioned variables along the interface. We use the continuum surface force (CSF) formulation [5] to represent the capillary forces as body forces by replacing the interface normal and the Dirac function in Eq. 4 by the gradient of the colour function,

$\mathbf{F}_{\text {cap }}=\sigma k \nabla \alpha$.

The curvature of the interface $k$ is computed from the colour function by,

$$
\begin{aligned}
k & =-\nabla \cdot \mathbf{n}_{\mathrm{I}}+\left(\mathbf{n}_{\mathrm{I}} \cdot \nabla \mathbf{n}_{\mathrm{I}} \cdot \mathbf{n}_{\mathrm{I}}\right) \\
& =-\nabla \cdot \frac{\nabla \alpha}{|\nabla \alpha|}+\left(\frac{\nabla \alpha}{|\nabla \alpha|} \cdot \nabla \frac{\nabla \alpha}{|\nabla \alpha|} \cdot \frac{\nabla \alpha}{|\nabla \alpha|}\right) .
\end{aligned}
$$

In the above equation, the first term is the conventional way to compute the interface curvature. The correction term (enclosed in brackets, available in OpenFOAM) ensures that the colour function $\alpha$ is interpolated accurately during the computation of the interface normal. It is formally zero but numerically not. The capillary pressure $p_{c}$ is computed solving the following equation [19],

$\nabla \cdot \nabla p_{c}=\nabla \cdot \mathbf{F}_{\text {cap }}$

The capillary pressure $p_{c}$ is solved using a Neumann condition $\left(\nabla p_{c}=0\right)$ and $\mathbf{F}_{\text {cap }}$ is computed from Eq. 5.
Finally, the colour function is advected in a conservative form with the help of the continuity equation, Eq. 1 as,

$\frac{\partial \alpha}{\partial t}+\nabla \cdot(\mathbf{U} \alpha)+\nabla \cdot\left(\alpha(1-\alpha) \mathbf{U}_{\mathrm{r}}\right)=0$.

$\mathbf{U}_{\mathrm{r}}$ in the above equation refers to the relative fluid velocity between phases acting at the interface approximated as $\min \left(\mathbf{U}_{\text {cell }}, \max \left(\mathbf{U}_{\text {domain }}\right)\right)$ [21].

\subsection{Boundary conditions on the wall}

We use an equilibrium contact angle $\theta$ based on Young's law [3] at the wall boundaries such that the unit normal to the interface (used in Eq. 6 to compute the interface curvature) is given by,

$\mathbf{n}_{\mathrm{I}}=\mathbf{n}_{\mathrm{w}} \cos (\theta)+\mathbf{t}_{\mathrm{w}} \sin (\theta)$,

where $\mathbf{n}_{\mathrm{w}}$ is the unit normal and $\mathbf{t}_{\mathrm{w}}$ is the unit tangent to the wall boundaries [5].

Displacing a meniscus on a no-slip wall boundary results in a mesh-dependent stress singularity on the wall surface $[1,11]$. To avoid this numerical artefact, we employ the partial (Navier) slip condition [17] such that the velocity on the wall boundaries is given by,

$U_{\mathrm{wall}}=\lambda \frac{\partial U}{\partial n}$,

where, $\lambda$ is the slip length and $\partial U / \partial n$ refers to the velocity gradient in the direction normal to the wall boundaries.

\subsection{Discretisation and numerical schemes}

We use the interFoam solver of the open-source finite volume based package - Foam-Extend-1.6 (http://wikki. gridcore.se) that employs the VOF-CSF formulation. A collocated Eulerian grid is used where the primary variables are stored at the cell centres of a control volume. An implicit first-order Euler scheme is employed for temporal discretisation. Gauss linear scheme is used for discretizing the gradient terms. Limited linear differencing is used to solve for the advection term in the momentum equation of NSEs. To adhere to the boundary limits of the VOF colour function, van Leer scheme is used to solve the advection equation of the colour function (Eq. 8). The primary variables, pressure and velocity are coupled by the predictor-corrector step of pressure implicit with splitting of operators (PISO) [12]. The time step size is restricted by the Brackbill number [6],

$\Delta t=\sqrt{\frac{\Delta x^{3} \rho_{\mathrm{avg}}}{\pi \sigma} .}$

The Courant number is set to 0.1 . 


\section{Transition zone shapes and the occurrence of capillary barrier zones}

In this section, we discuss the shape of the synthetic $2 \mathrm{D}$ pore geometry under investigation, the threshold capillary pressures used by conventional quasi-static PNM for the considered pore geometry and the concept of capillary barriers in detail.

\subsection{Pore geometry}

We consider a synthetic two-dimensional pore geometry where the shape of the transition zone in dimensionless form is defined by the function

$\bar{y}=s \cdot \frac{\left(\frac{\bar{x}}{s}\right)^{n}}{\left(\frac{\bar{x}}{s}\right)^{n}+\left(1-\frac{\bar{x}}{s}\right)^{n}}+0.5$, with0 $\leq \frac{\bar{x}}{s} \leq 1$.

In the above equation, $s$ is a scaling factor to tune the radius of the pore body $\mathrm{r}_{\mathrm{p}}$ and $n$ is an exponent to tune the shape of transition zone. The dimensionless variables $\bar{x}=x / d$ and $\bar{y}=y / d$ are obtained by dividing $x, y$ by the reference length, $d=2 r_{\mathrm{t}}=20 \mu \mathrm{m}$. The reference length is taken as the diameter of the pore throat. The shape of the transition zone for different values of $n$ is illustrated in Fig. 1. As $n$ increases, the transition zone gets sharper. The radius of the pore throat is set to $\mathrm{r}_{\mathrm{t}}=d / 2=10 \mu \mathrm{m}$ all through the manuscript. The aspect ratio of the pore geometry is defined as $\mathrm{AR}=\mathrm{r}_{\mathrm{p}} / \mathrm{r}_{\mathrm{t}}=\left(\mathrm{r}_{\mathrm{t}}+s d\right) / \mathrm{r}_{\mathrm{t}}$. The aspect ratio is three for the pore geometry illustrated in Fig. 1 having a scaling factor, $s=1$. The value of the scaling factor is mentioned when it is not equal to one. The slope of the wall boundary in the transition zone $m_{w}$ is maximum at the centre ( $\mathrm{x}=10 \mu \mathrm{m}$, $\mathrm{y}=\mathrm{r}_{\mathrm{t}}+s d / 2=20 \mu \mathrm{m}$ ) equal to $n$ and decreases to zero at the entrance and exit of the transition zone as illustrated in Fig. 3b.

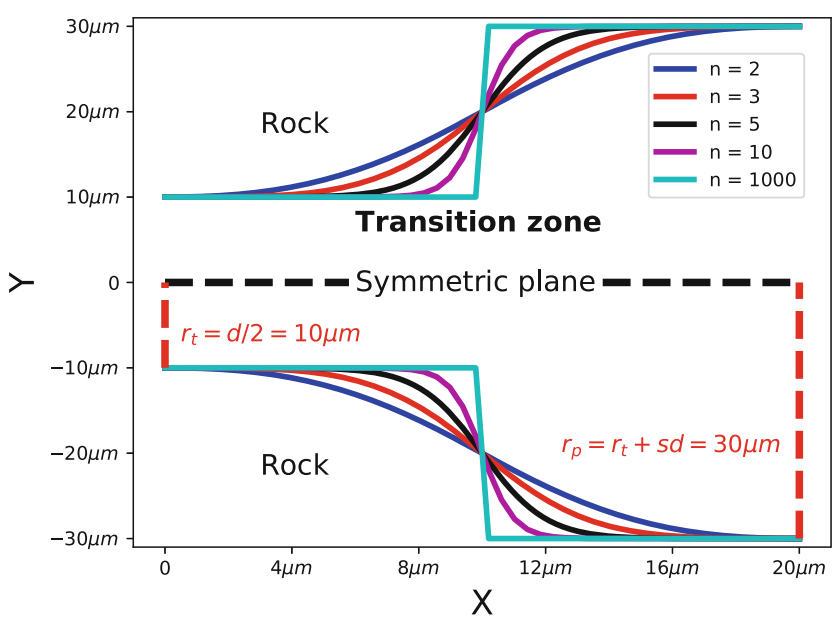

Fig. 1 Illustration of the shape of transition zones connecting a pore throat with a pore body

\subsection{Threshold capillary pressures}

Provided that the topological information of porous media is known, PNM construct a network model of pore spaces considering simplified geometries to represent the real pore spaces. Typically, the throats are represented as cylinders and pore bodies are represented as spheres [7]. For the considered synthetic pore geometry discussed in Section 3.1, PNM generally consider the pore throat as a rectangle and the pore body as a circle as illustrated by blue line in Fig. 2. Conventional quasi-static PNM rely on the YoungLaplace law (Eq. 13, [3]) to decide the invasion pattern of fluids in a system

$p_{t}=\sigma k=\sigma\left(\frac{1}{r_{1}}+\frac{1}{r_{2}}\right)$

where $p_{t}$ is the threshold capillary pressure and $r_{1}, r_{2}$ are the principal interfacial radii. The critical radius of the interface that corresponds to the least interfacial curvature encountered during the invasion process is used to compute the threshold capillary pressure using Eq. 13. The threshold capillary pressure is greater for smaller pores. Pore spaces are invaded by the wetting phase when the available pressure gradient is lower than the threshold capillary pressure [14]. Hence, smaller pores are preferred during imbibition. However, the quantitative predictions of the threshold capillary pressure that PNM use to decide if a pore gets invaded are still approximate [4]. PNM predict the imbibition path in a throat-pore body system according to favourable threshold capillary pressures encountered. During the network extraction of PNM, the shape of transition zone is not taken into account as illustrated in Fig. 2.

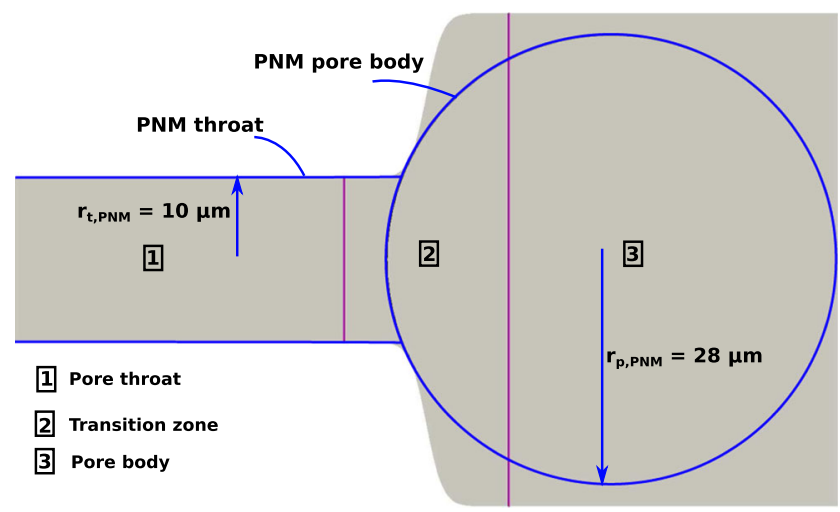

Fig. 2 Illustration of the pore geometry with the transition zone defined by $n=5$ (labelled 2) connecting the pore throat (labelled 1) and the pore body (labelled 3 ). Pore network extraction simplify this pore geometry into a rectangular throat having a radius of $10 \mu \mathrm{m}$ and a circular pore body having a radius of approximately $28 \mu \mathrm{m}$. In the conventional network extraction of PNM, the shape of the transition zone is neglected 
For the case shown in Fig. 2, for a contact angle $\theta=10^{\circ}$, the threshold capillary pressures in the throat and pore body are $984 \mathrm{~Pa}$ and $351 \mathrm{~Pa}$ respectively. The threshold capillary pressure for the pore body is defined considering that the critical radius $r$ in Eq. 13 is equal to $\cos (\theta) / r_{p, P N M}$, where $r_{p, P N M}$ is the PNM estimate of the pore body radius approximately equal to $28 \mu \mathrm{m}$. Similarly, when the contact angle is $\theta=13^{\circ}$, the threshold pressures in the throat and pore body are $974 \mathrm{~Pa}$ and $348 \mathrm{~Pa}$ respectively. We will discuss the threshold capillary pressure values attained using DNS considering the transition zone shape for both the discussed contact angles later in Section 5.1.

\subsection{Capillary barriers and critical contact angle}

This section discusses in detail the conditions responsible for the occurrence of capillary barrier zones during imbibition and their potential consequences on the flow dynamics. The capillary barrier zones are exclusively dependent on the wetting properties of the fluids and the shape of the transition zone. It is important to investigate the parameters that result in capillary barrier zones as their presence can potentially hinder the imbibition of a pore body thereby influencing the invasion paths. Theoretical analysis in this section is done considering spontaneous imbibition flow conditions and assuming that the system is occupied by light fluids (such as air) to neglect inertial effects.

The critical contact angle $\theta_{c}$ is a parameter exclusively dependent on the shape of the transition zone wall boundary. It is defined as the contact angle of the wetting phase $\theta$ that results in a flat meniscus at the centre of the transition zone (where the wall slope is maximum) as illustrated in Fig. 3a. Spontaneous imbibition flows are capillary driven. A flat meniscus corresponds to zero capillary forces and thus hinders further imbibition of the wetting phase into the pore body. We now present an analytical expression to determine $\theta_{c}$ for the considered synthetic pore geometry. Consider an infinitesimally small section at the centre of the transition zone as illustrated by the triangular region in
Fig. 3a. $\theta_{c}$ is the angle between the flat meniscus and the tangent drawn from the midpoint of the transition zone. $\gamma$ is the angle between the tangent drawn from the midpoint of the transition zone and a horizontal plane. The slope of the wall boundary $m_{w}$ is given by

$\tan (\gamma)=m_{w}=\frac{n(\bar{x})^{n-1}(1-\bar{x})^{n}+n(\bar{x})^{n}(1-\bar{x})^{n-1}}{\left(\bar{x}^{n}+(1-\bar{x})^{n}\right)^{2}}$

In the above expression, $x=10 \mu \mathrm{m}$ is the known location of the occurrence of a flat meniscus and $d=20 \mu \mathrm{m}$ is the reference length resulting in $\bar{x}=0.5$. As $\theta_{c}=\pi / 2-\gamma$ and $\gamma=\operatorname{atan}\left(m_{w}\right)$

$\theta_{c}=\frac{\pi}{2}-\operatorname{atan}\left(\frac{n(\bar{x})^{n-1}(1-\bar{x})^{n}+n(\bar{x})^{n}(1-\bar{x})^{n-1}}{\left(\bar{x}^{n}+(1-\bar{x})^{n}\right)^{2}}\right)$.

Substituting $\bar{x}=0.5$ in the above expression and after few arithmetic manipulations results in a simple analytic expression to determine $\theta_{c}$ given by,

$\theta_{c}=\frac{\pi}{2}-\operatorname{atan}(n)$

Figure 4a shows the critical contact angle for different values of $n$. As the transition zone gets sharper (increase of $n$ ), the critical contact angle gradually moves towards $0^{\circ}$ (at $n=\infty, \theta_{c}=0^{\circ}$ ).

The wetting properties of the fluid in relation to critical contact angle determine if the wetting phase can imbibe a pore body. Figure 4 illustrates the possible scenarios. The first scenario is when $\theta=\theta_{c}$. This results in the occurrence of a flat meniscus at the centre of the transition zone as discussed above (Fig. 3a). The second scenario is when $\theta<\theta_{c}$. This always favours imbibition as the contact angle of the wetting phase is sufficient to maintain a

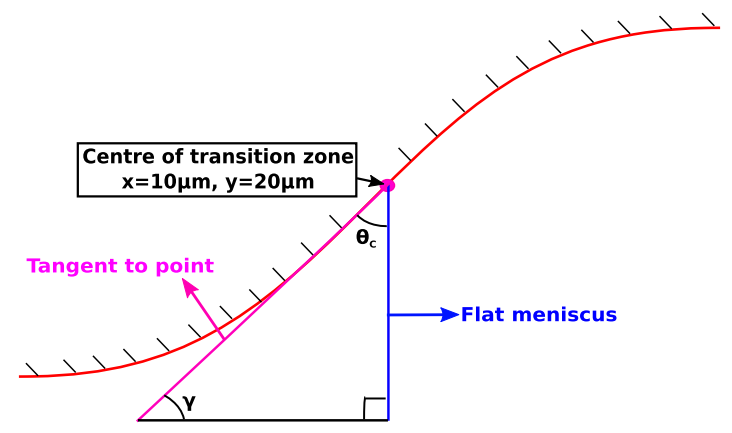

(a)

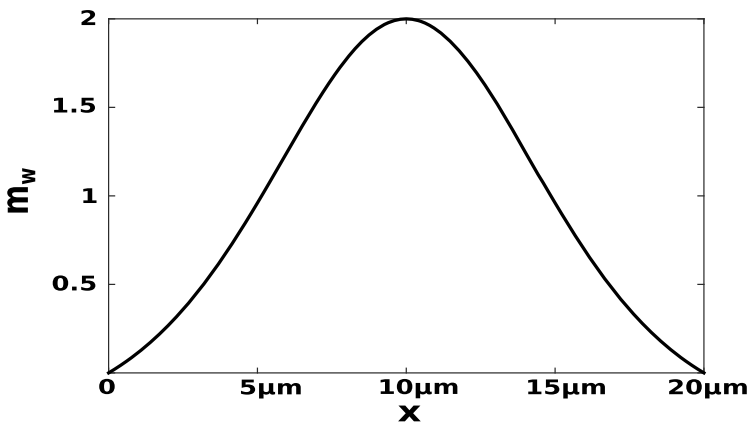

(b)

Fig. 3 a Illustration of the critical contact angle $\theta_{c}$ which is the contact angle of the wetting phase that results in the occurrence of a flat meniscus at the centre of the transition zone $(\mathrm{x}=10 \mu \mathrm{m}, \mathrm{y}=20 \mu \mathrm{m})$. $\mathbf{b}$ Illustration of the slope of wall boundary $m_{w}$ in the transition zone for $n=2$ 


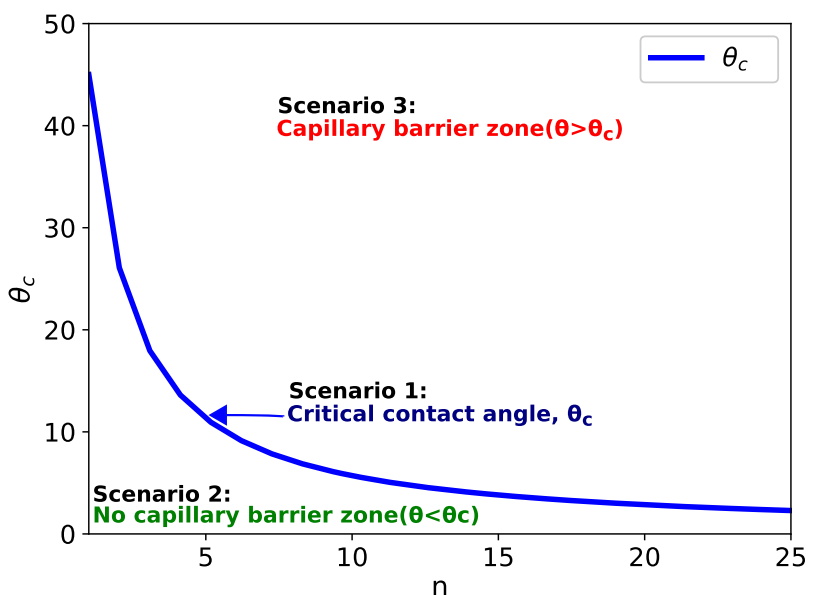

(a)

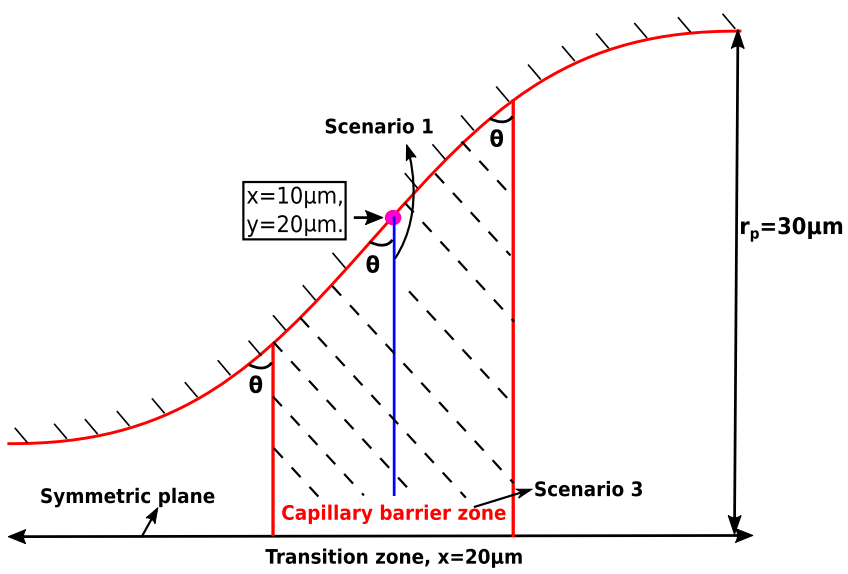

(b)

Fig. 4 a Critical contact angle with respect to $n$ and classification of the imbibition process based on $\theta$ with respect to $\theta_{c}$. $\mathbf{b}$ Meshed region illustrates the capillary barrier zone when $\theta>\theta_{c}$

meniscus curvature (and subsequently the capillary driving forces) at all times. The final scenario is when $\theta>$ $\theta_{c}$. This results in the occurrence of two equidistant flat menisci from the centre of the transition zone. We refer to the region enclosed between the two flat menisci as the capillary barrier zone (Fig. 4b). The significance of capillary barrier zone is illustrated in Fig. 5 which shows the meniscus orientation at different positions of the pore space. Within the capillary barrier zone, the meniscus orients with a negative curvature resulting in capillary forces acting against imbibition. Transitions in the meniscus curvature during forced imbibition were investigated by Ferrari and Lunati [8] on pores having corners with singularity (corners of a sharp pore body has an undefined wall normal). They refer to the transitions in meniscus curvature occurring at the corners of a pore body as meniscus reconfigurations. The transition in meniscus curvature during quasi-static imbibition in a single junction micromodel were also observed experimentally [25]. Zacharoudiou et al. [25] used Lattice-Boltzmann simulations to investigate spontaneous imbibition in a sharp throat-pore body system. From their numerical investigation, it was noticed that the interfacial oscillations do occur even for spontaneous imbibition flows. However, in all of their investigated cases, the wetting phase always invaded the pore space. As illustrated in Fig. 5, the meniscus changes its curvature during imbibition even when there is a smooth transition between a throat and a pore. The transitions in meniscus curvature are dependent on the wetting properties of the fluid and the shape of the wall boundary.

Now, we determine the position of the capillary barrier zones when $\theta>\theta_{c}$ for the considered synthetic pore geometries. From Fig. 3a,

$$
\tan (\gamma)=\tan \left(\frac{\pi}{2}-\theta\right)
$$

Fig. 5 Illustration of the meniscus configurations at different positions of the pore space. Within the capillary barrier zone, capillary forces act as resisting forces against imbibition

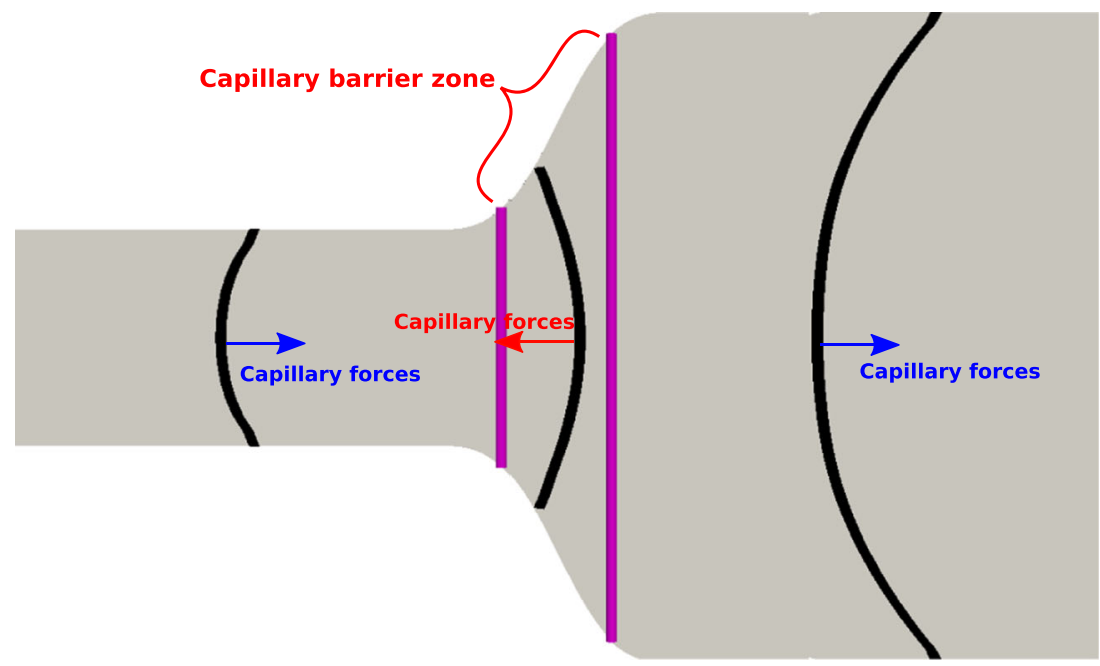


Substituting $m_{w}$ from Eq. 14 in the above expression for $\tan (\gamma)$ gives,

$$
\frac{n(\bar{x})^{n-1}(1-\bar{x})^{n}+n(\bar{x})^{n}(1-\bar{x})^{n-1}}{\left(\bar{x}^{n}+(1-\bar{x})^{n}\right)^{2}}=\tan \left(\frac{\pi}{2}-\theta\right) .
$$

In the above polynomial expression, for a known shape of the transition zone $n$ and contact angle of the wetting phase $\theta$, the real roots of $\bar{x}$ are computed using [15] to determine the limits of the capillary barrier zones. Figure 6 shows the normalised width of the capillary barrier zones and the critical contact angles (marked by stars on the horizontal axis) for different $n$. We arrive at two important conclusions from Fig. 6. Firstly, for any $n$, the width of the capillary barrier zone $x^{\prime}$ increases with the contact angle $\left(x^{\prime} \propto \theta\right)$. Secondly, the width of the barrier zone reduces at larger $\theta$ and $n\left(x^{\prime} \propto 1 / n\right)$ corresponding to a decrease in the width of the transition zone where the wall slope varies for larger $n$ (Fig. 1).

We refer to the discussed barrier zones as dynamic capillary barriers as during spontaneous imbibition, inertia can assist the wetting phase to overcome the capillary barriers. During this phase, the meniscus is subjected to smooth transitions in its curvature multiple times. The first transition in meniscus curvature occurs at the entrance of the barrier zone where a positive meniscus curvature (exists until the meniscus is behind the entrance of the barrier zones) turns negative (exists in barrier zones). When the meniscus is in capillary barrier zones, capillary forces accompany viscous forces to resist further spontaneous imbibition. If inertia is dominant enough to overcome resisting forces, the meniscus is pushed over capillary barrier zones where the meniscus curvature transitions back from negative to positive (Fig. 5).

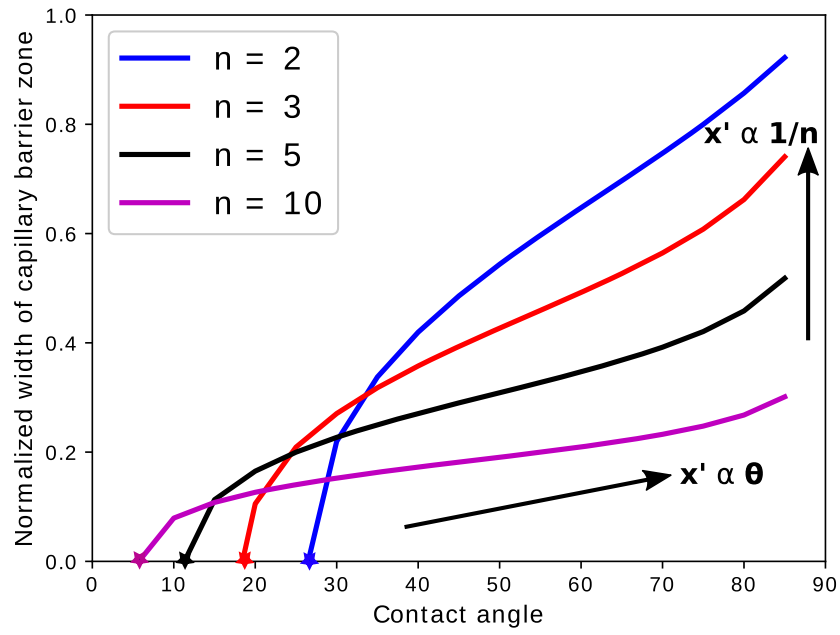

Fig. 6 Normalised width of capillary barrier zone $x^{\prime}$ with respect to $n$. The stars on the horizontal axis represent the occurrence of one flat meniscus at the centre of the transition zone when $\theta=\theta_{c}$
In addition to the capillary barrier concept discussed above, there also exists a barrier contact angle $\theta_{b}$ that depends on the fluid properties, contact angle and the pore geometry. We determine the barrier contact angle numerically for different inertial systems by considering different fluid densities in the pore space but maintain the surface tension between fluids and viscosity of fluids as constants. For dense fluids, $\theta_{b}$ is notably larger than $\theta_{c}$; whereas for light fluids, $\theta_{b}$ is slightly greater than $\theta_{c}$. For $\theta_{c} \leq \theta \leq \theta_{b}$, inertia assists the wetting phase to overcome the resisting forces thereby invading the pore space. On the other hand, for $\theta>\theta_{b}$, the resisting forces are greater than inertia and the wetting phase cannot overcome the capillary barrier zones and hence cannot imbibe the pore space.

\section{Numerical setup}

In this section, the mesh, initial and boundary conditions used for the investigation of water flooding with spontaneous imbibition flow conditions are discussed. These parameters are then used in Section 5 to study the contact angle dependent invasion patterns.

\subsection{Computational domain}

The pore geometries used for investigation in this manuscript along with the meshing procedure are provided in the Supplementary Material. A two-dimensional Cartesian mesh with refinement $\Delta x=\Delta y=0.5 \mu \mathrm{m}$ is embedded into the modelled pore geometries to snap off the hexahedral Cartesian control volumes such that the pore shape is captured accordingly using Foam-Extend's library snappyHexMesh. Figure 7 illustrates the mesh and spontaneous imbibition boundary conditions used for investigating flow through a single pore body having an aspect ratio of 3 . The length of the pore throat and the pore body is $2 x$ ( $x=$ $20 \mu \mathrm{m}$ ) and the length of the transition zone is $x$.

All simulations were performed on 4 Intel-Xeon processors $(2.000 \mathrm{GHz}$ frequency) and run until the wetting phase enters the pore body (if imbibing) else until no change in the volume of the wetting phase occurs (if stopped by capillary barriers). When the density of fluids considered are small $\left(\rho_{\alpha}=1 \mathrm{~kg} / \mathrm{m}^{3}\right)$ the computation time was around $6 \mathrm{~h}$ using a Brackbill number $5\left(\Delta t=10^{-8} \mathrm{~s}\right)$. While considering heavier fluids (example: $\rho_{\alpha}=1000 \mathrm{~kg} / \mathrm{m}^{3}$ ), the computation times were within 20 min using Brackbill number one $\left(\Delta t=6.3 \times 10^{-8} \mathrm{~s}\right)$.

\subsection{Initial and boundary conditions}

We initially position the meniscus $10 \mu \mathrm{m}$ from the inlet boundary and allow the meniscus to relax for $0.1 \mathrm{~ms}$ 


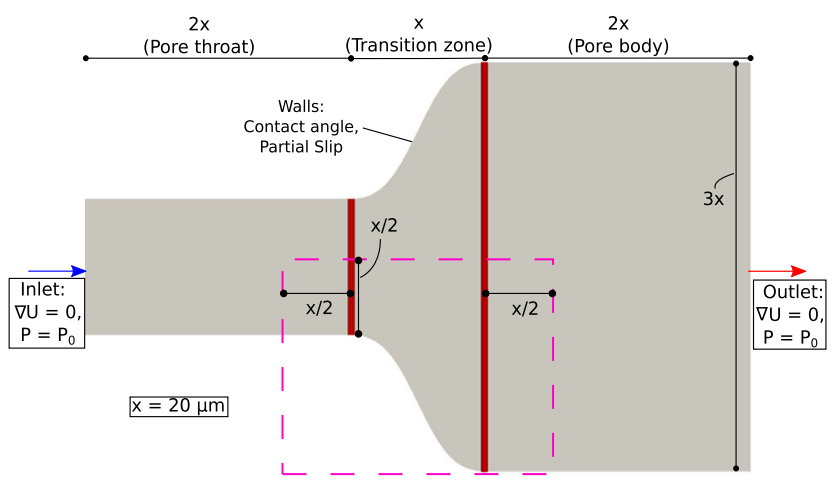

(a)

Fig. 7 Numerical setup for investigating spontaneous imbibition in a pore singlet. a The pore space comprises of the pore throat, transition zone and pore body. The boundary conditions for the inlet, outlet and walls are shown. b A zoom of the meshed pore space highlighted by

to develop an initial meniscus curvature. During the relaxation stage, the pressure on the boundaries is set to Neumann condition $(\nabla p=0)$ and there is neither inflow nor outflow across the boundaries $(\mathbf{U}=0)$. This results in the relaxation of the meniscus according to the contact angle boundary condition Eq. (9). Then we commence spontaneous imbibition by using a Dirichlet boundary condition for the pressure $\left(p_{\text {inlet }}=p_{\text {outlet }}=\right.$ $\left.p_{0}\right)$ and a Neumann boundary condition for the velocity $(\nabla \mathbf{U}=0)$ at the inlet and outlet (Fig. 7a). The flow with spontaneous imbibition conditions is a consequence of the pressure gradient developed within the pore geometry due to capillarity [18].

To alleviate the numerical artefact of stress singularity on the wall boundaries caused due to a moving contact line on a no-slip boundary, a partial slip wall boundary is chosen considering uniform slip length of $\lambda=2 \mu \mathrm{m}$. The slip length is chosen such that we resolve the size of control volumes in the computational domain [1].

\section{Results}

In this section, we systematically investigate the principles of pore body filling under different inertial conditions and then extend the study to a simple pore network model (a pore doublet) comprising of two pores having different size and shape. For all the cases discussed in this section, the surface tension between wetting and non-wetting fluids is set to $\sigma=0.01 \mathrm{~N} / \mathrm{m}$ and the viscosity of both fluids is taken to be $\mu=0.001 \mathrm{~kg} / \mathrm{ms}$. The density and contact angles for the cases discussed vary and are mentioned. The density and viscosity ratios are set to unity. Gravitational effects have been discarded in this investigation considering the

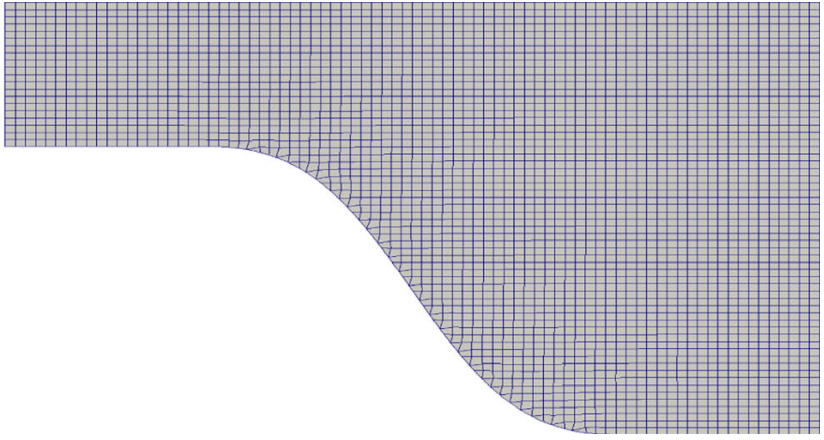

(b)

the dashed pink region in subfigure (a). The control volumes are Cartesian except near the transition zone wall which are adjusted to match the shape of the wall

size of the pore geometry (in microns) and as the fluids in the system have no density differences. The animations of all the contact angle dependent invasions investigated in this manuscript are provided in the Supplementary Material.

\subsection{Spontaneous imbibition through a single pore body}

We consider the pore space comprising of a single pore throat, transition zone and pore body as illustrated in Fig. 7. We commence this study by analysing the invasion patterns when inertial effects are negligible and later consider systems where inertia does play a role during the invasion process.

\subsubsection{Weak inertial system}

The density of both fluids in the system is $1 \mathrm{~kg} / \mathrm{m}^{3}$ (air). This case study corresponds to the theoretical concept put forward in Section 3 where there are no additional forces acting within the system apart from capillary and viscous forces that govern the flow physics. However, it is important to note that it is rather difficult to have two immiscible fluids at such low densities in real-life applications. We validate cases with the transition zone wall shapes defined by $n=2,3,5,7$ and 10 (Eq. 12, Fig. 1). For discussion, we consider $n=5$ and the meniscus dynamics for other cases are qualitatively similar.

For weak inertial systems, after the initial relaxation stage of the meniscus, when spontaneous imbibition commences, the meniscus is displaced at a steady-state velocity within the pore throat according to the balance between capillary (driving force) and viscous (resisting) forces. The instantaneous steady-state velocity is a consequence of the minimal mass within the system to be displaced. 
The variation of wall slope along the transition zone (Fig. 3b) in relation with the contact angle of the wetting phase impacts the meniscus curvature and correspondingly the capillary forces and imbibition rates. As the wall slope is maximum at the centre of the transition zone and reduces in both directions moving away from the centre, the most difficult stage of the spontaneous imbibition process is to overcome the centre of the transition zone where the meniscus curvature decreases resulting in a slow down of the meniscus displacement if $\theta<\theta_{c}$. On the other hand, for $\theta>\theta_{c}$, there is no meniscus curvature at the entrance of the capillary barrier zone thereby hindering further imbibition of the wetting phase into the pore body.
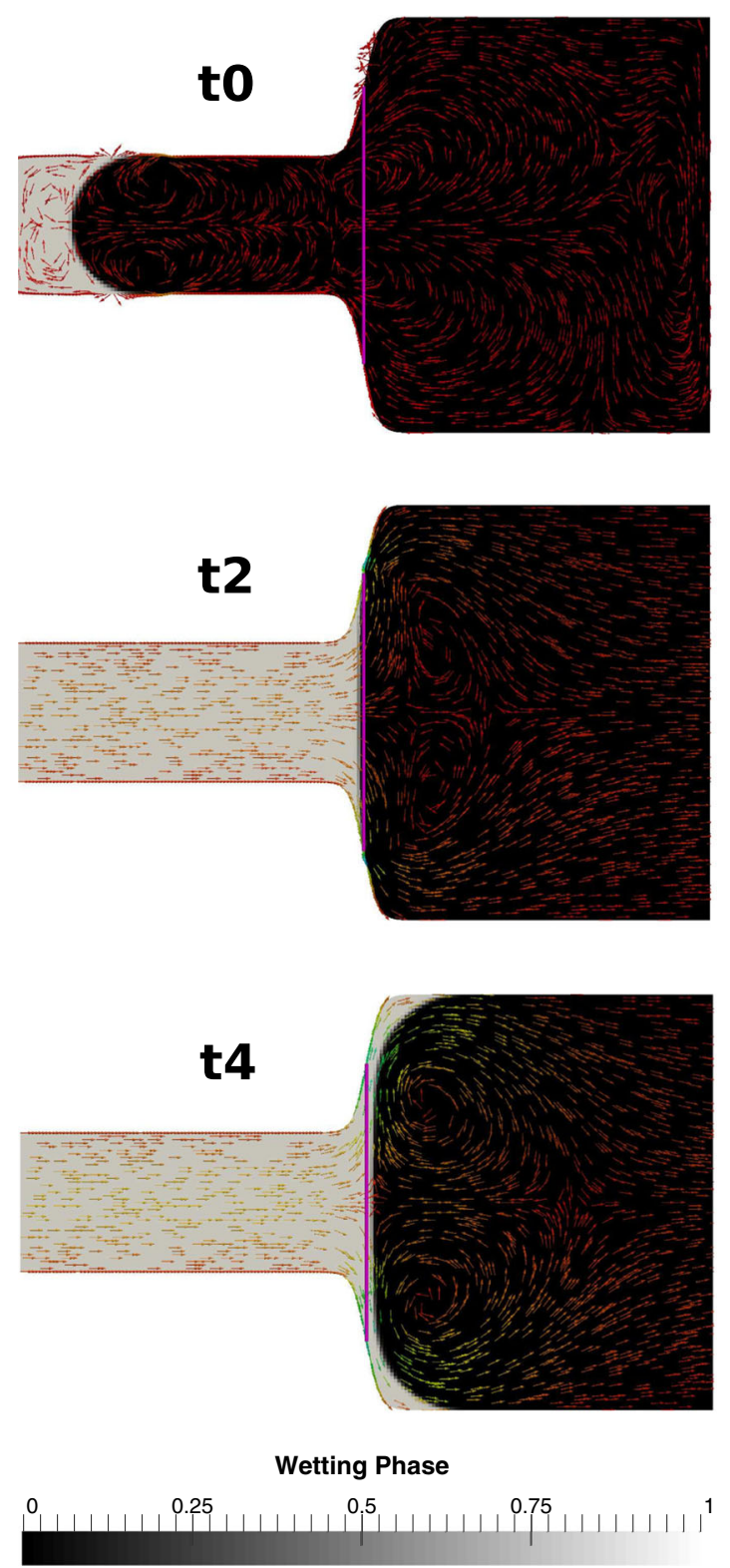

Figure 8 shows the snap-shots of the meniscus position at different time intervals when the contact angle of the wetting phase is $\theta=10^{\circ}$ which is less than the critical contact angle $\theta_{c}=11.31^{\circ}$. The pink solid line represents the centre of the transition zone for reference. The orientation of the velocity vectors is shown to highlight the flow behaviour. At $t 0=0.1 \mathrm{~ms}$, the meniscus reaches an equilibrium configuration according to the contact angle boundary conditions. This is the end of the relaxation stage from where we commence spontaneous imbibition. At $t 1=0.15 \mathrm{~ms}$, the meniscus enters the transition zone. The displacement velocity of the meniscus commences to reduce from the steady-state velocity attained when the
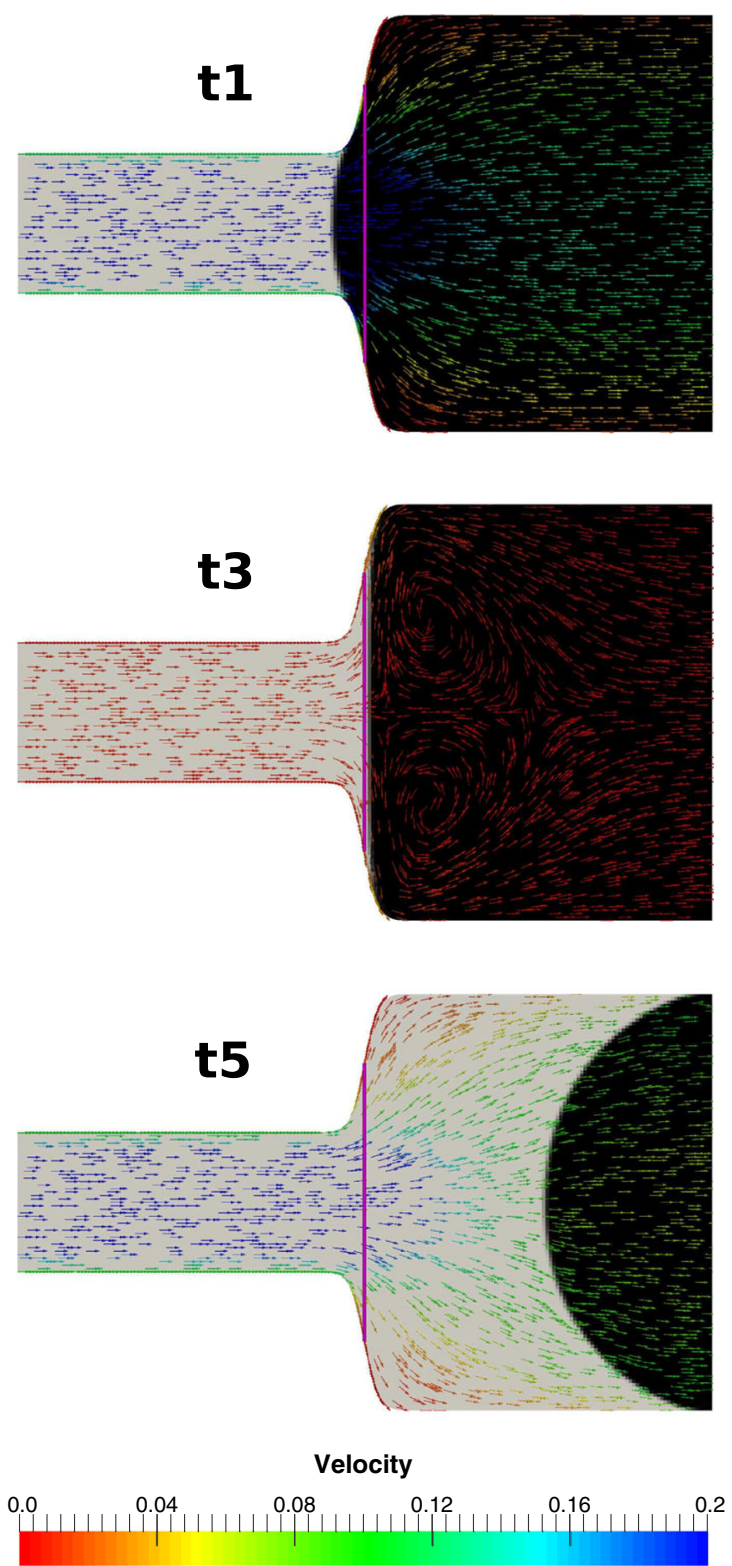

Fig. 8 Spontaneous imbibition for $n=5, \rho_{\alpha}=1 \mathrm{~kg} / \mathrm{m}^{3} \cdot \theta\left(10^{\circ}\right)<\theta_{c}\left(11.31^{\circ}\right)$. The corresponding time related to the snapshots are $t 0=0.1 \mathrm{~ms}$, $t 1=0.15 \mathrm{~ms}, t 2=0.2 \mathrm{~ms}, t 3=0.6 \mathrm{~ms}, t 4=0.9 \mathrm{~ms}$ and $t 5=1.4 \mathrm{~ms}$ 

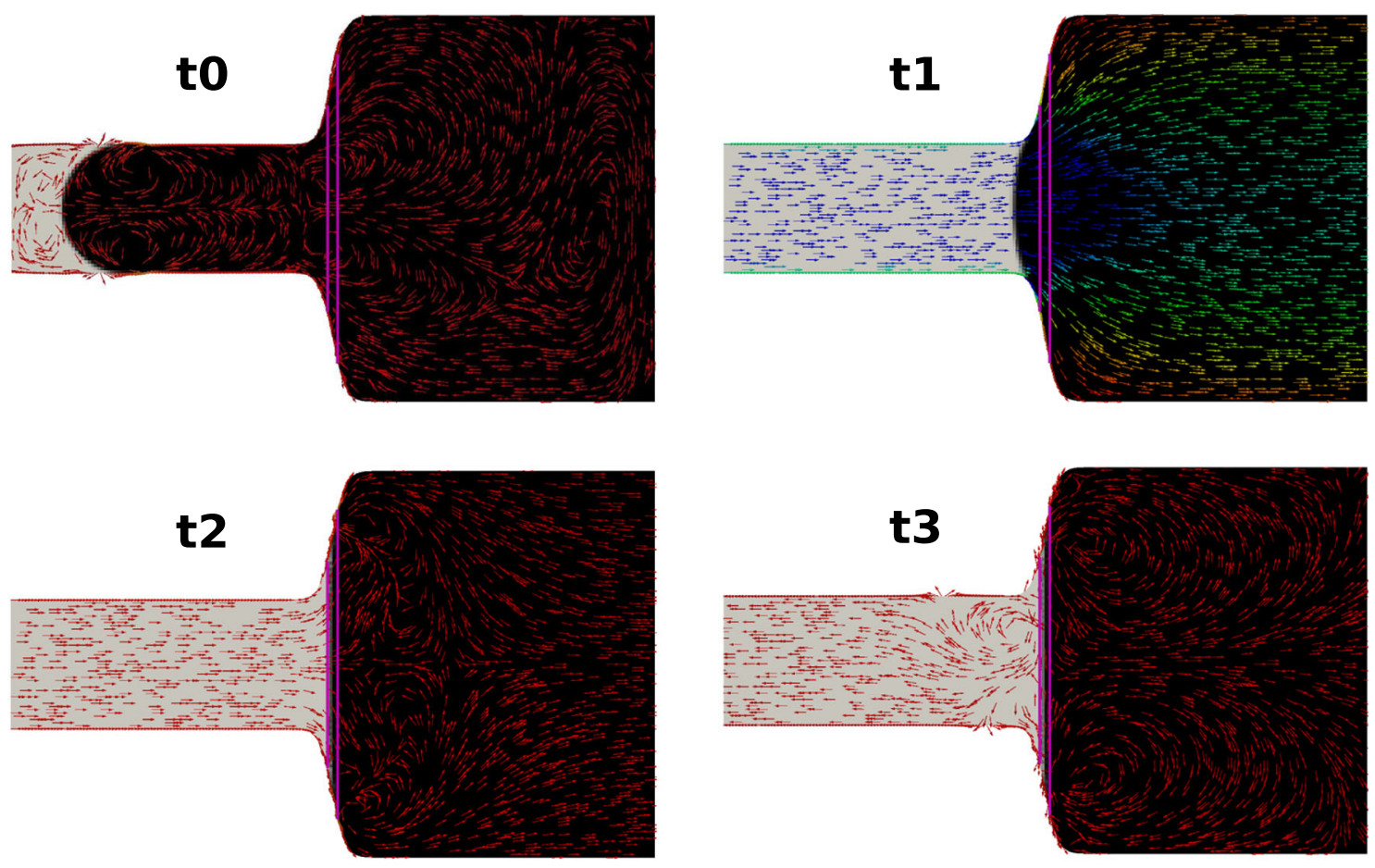

Wetting Phase
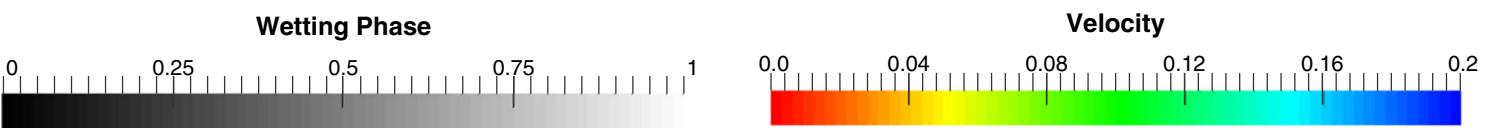

Fig. 9 Spontaneous imbibition for $n=5, \rho_{\alpha}=1 \mathrm{~kg} / \mathrm{m}^{3} . \theta\left(13^{\circ}\right)>\theta_{c}\left(11.31^{\circ}\right)$. The corresponding time related to the snapshots are $t 0=0.1 \mathrm{~ms}$, $t 1=0.15 \mathrm{~ms}, t 2=0.3 \mathrm{~ms}$ and $t 3=3 \mathrm{~ms}$

meniscus was present in the pore throat due to the decrease in meniscus curvature. At $t 2=0.2 \mathrm{~ms}$, as the wetting phase imbibes further and reaches the centre of the transition zone, the imbibition rate further drops due to decrease in the meniscus curvature. At $t 3=0.6 \mathrm{~ms}$, the meniscus slowly overcomes the centre of the transition zone (having maximum wall slope) and commences to enter a favourable zone to imbibe due to the continuous reduction in the wall slope. However, the imbibition of the wetting phase is slow due to smaller meniscus curvature caused by the nature of the expanding pore geometry. At $t 4=0.9 \mathrm{~ms}$, the meniscus exits the transition zone and starts to equilibrate towards a new meniscus configuration based on the contact angle boundary conditions thereby generating vortices. Finally, at $t 5=1.4 \mathrm{~ms}$, the wetting phase keeps filling the pore body at a steady-state velocity based on the balance between capillary and viscous forces.

Figure 9 shows the snap-shots of the meniscus position at different intervals of time when the contact angle of the wetting phase is $\theta=13^{\circ}$ which is greater than the critical contact angle $\theta_{c}=11.31^{\circ}$. From Section 3, for this scenario, we show that there exists a capillary barrier zone and the position of the barrier zone can be found semi-analytically from Eq. 18. The region enclosed between two pink lines in Fig. 9 represents the capillary barrier zone. At $t 0=0.1 \mathrm{~ms}$, the meniscus reaches an equilibrium configuration according to the contact angle boundary conditions at the end of the relaxation stage from where we start spontaneous imbibition. At $t 1=0.15 \mathrm{~ms}$, we observe the meniscus entering the transition zone and the meniscus velocity starts to reduce from the steadystate velocity attained when the meniscus was present in the pore throat due to reduction in the meniscus curvature as explained for the previous case. At $t 2=0.3 \mathrm{~ms}$, the meniscus slows down at the entrance of the capillary barrier zone due to the flat meniscus configuration. As explained in Section 3, a flat meniscus would mean zero capillary forces thereby hindering further spontaneous imbibition into the pore body. Finally, at $t 3=3 \mathrm{~ms}$, a long time after the wetting phase has reached the entrance of the capillary barrier zone, we observe no substantial difference in the wetting phase occupancy compared to $t 2$.

Figure 10 shows the evolution of the capillary pressure during spontaneous imbibition for both the above discussed cases. The difference in the computed capillary pressure values Eq. 7 between the outlet and inlet is plotted as capillary pressure (coloured dots) in Fig. 10. From Fig. 10 when $\theta<\theta_{c}$, the meniscus curvature stays positive all through the spontaneous imbibition process and the threshold capillary pressure is a positive value (represented 


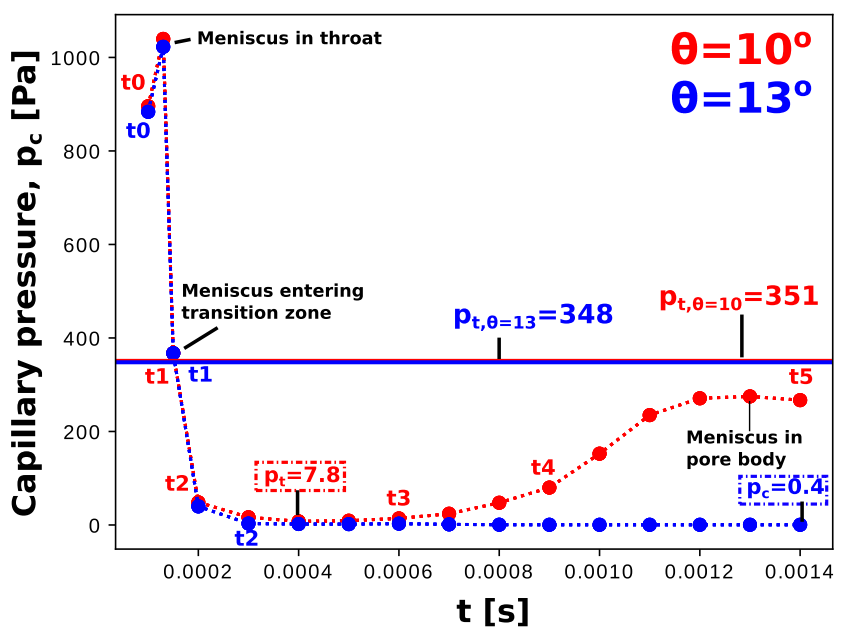

Fig. 10 Evolution of the capillary pressure during spontaneous imbibition in a pore geometry having transition zone defined by $n=5$. The red dots represent capillary pressure values for $\theta=10^{\circ}$ and blue dots represent capillary pressure values for $\theta=13^{\circ} . t x$ correspond to the meniscus configurations shown in Figs. 8 and 9. Threshold capillary pressure for $\theta=10^{\circ}$ is $p_{t}=7.8 \mathrm{~Pa}$ and for $\theta=13^{\circ}$ is a negative value which are notably different compared to the values used in conventional PNM

by red dots). Interestingly, we find that the threshold capillary pressure for the system is $p_{t}=7.8 \mathrm{~Pa}$ which is much lesser than the value used by PNM $\left(p_{t}=351 \mathrm{~Pa}\right)$. Note that we find discrepancy in the numerically determined capillary pressure values and the values obtained by YoungLaplace law (for example: $\theta=10^{\circ}$ case) when the meniscus is in the throat (numerical prediction of $1039 \mathrm{~Pa}$ against 985 $\mathrm{Pa}$ ) and pore body (numerical prediction of $275 \mathrm{~Pa}$ against $328 \mathrm{~Pa}$ ) due to the dynamic nature of meniscus displacement occurring at high velocities. For the case $\theta>\theta_{c}$, the capillary pressure (represented by blue dots) is zero due to flat meniscus configuration that occurs at the entrance of the capillary barrier zone. Hence, the meniscus is unable to invade the pore space any further. As shown in Fig. 5, when the meniscus enters the capillary barrier zone, the meniscus curvature transforms from positive (imbibition curvature) to negative (drainage curvature). This indicates that the threshold capillary pressure when $\theta>\theta_{c}$ is negative for the considered pore body which means an additional push is required for the meniscus to overcome the capillary barrier zone. Interestingly, due to the negative threshold capillary pressure encountered when $\theta>\theta_{c}$, the phenomena can be related to fluid displacement in a mixed-wet system where the pores having capillary barriers have inverted their wettability.

When spontaneous imbibition is stopped due to capillary barrier effects, we compare the numerical solution of the volume of wetting phase present within the pore geometry with the expected volume to be present within the pore geometry. The discrepancy is always within $5 \%$ for all the cases investigated in this paper. For the case discussed $(\mathrm{n}=5$, $\left.\theta\left(13^{\circ}\right)>\theta_{c}\left(11.31^{\circ}\right)\right)$, the discrepancy is around $2.1 \%$ potentially caused due to numerical dispersion.

\subsubsection{Dynamic imbibition of a pore body-role of inertia}

We now consider the same numerical setup as discussed above but consider the impact of inertia in analysing the invasion patterns. Both the fluids in the system have a density of $1000 \mathrm{~kg} / \mathrm{m}^{3}$ (water). We investigated the spontaneous imbibition process on wall shapes defined by $n=2,3,5,7$ and 10. For discussion, we consider $n=$ 10 and the flow behaviour is qualitatively similar for the other investigated cases. An inertial dominant system would take longer duration to reach steady state as the mass to be displaced is greater compared to a system occupied by lighter fluids. Therefore, compared to the previous case $\left(\rho_{\alpha}=1 \mathrm{~kg} / \mathrm{m}^{3}\right)$ where steady-state velocity is attained instantaneously on starting spontaneous imbibition, for an inertial dominant system, the flow gradually accelerates towards steady state. Considering the flow within the system carrying sufficient momentum to overcome the capillary barrier zones, the meniscus curvature smoothly transforms twice. The first meniscus curvature transition occurs at the entrance of the capillary barrier zone where the imbibition curvature (positive curvature) turns to a drainage curvature (negative curvature). The second meniscus curvature transition occurs at the exit of the capillary barrier zone where the drainage curvature turns backs to an imbibition curvature. When the meniscus is being displaced within the capillary barrier zone, capillary forces accompany viscous forces to resist further spontaneous imbibition. Even during this stage, the slowing down of the spontaneous imbibition process is gradual. Therefore, inertia can temporarily assist the wetting phase to overcome the capillary barrier zones thereby invades the pore body.

Figure 11 shows the snap-shots of the meniscus position over time for the transition zone shape defined by $n=10$ when the contact angle of the wetting phase is $\theta=38^{\circ}$ which is larger than the critical contact angle $\theta_{c}=5.71^{\circ}$. For this case, the fluid system carries sufficient momentum to overcome the capillary barrier zone. Note that $\theta=38^{\circ}$ is at the brink of overcoming the barrier zone and imbibes the pore space; whereas for $\theta=39^{\circ}$ (discussed later), the inertia of the system is not sufficient to overcome resisting forces and push the meniscus over the capillary barrier zone. The region enclosed between two solid pink lines represent the capillary barrier zone determined semianalytically. The orientation of the velocity vectors is shown to analyse the flow behaviour. At $t 0=0.1 \mathrm{~ms}$, the meniscus reaches an equilibrium configuration according to the contact angle boundary conditions from where we commence spontaneous imbibition. At $t 1=0.2 \mathrm{~ms}$, the 

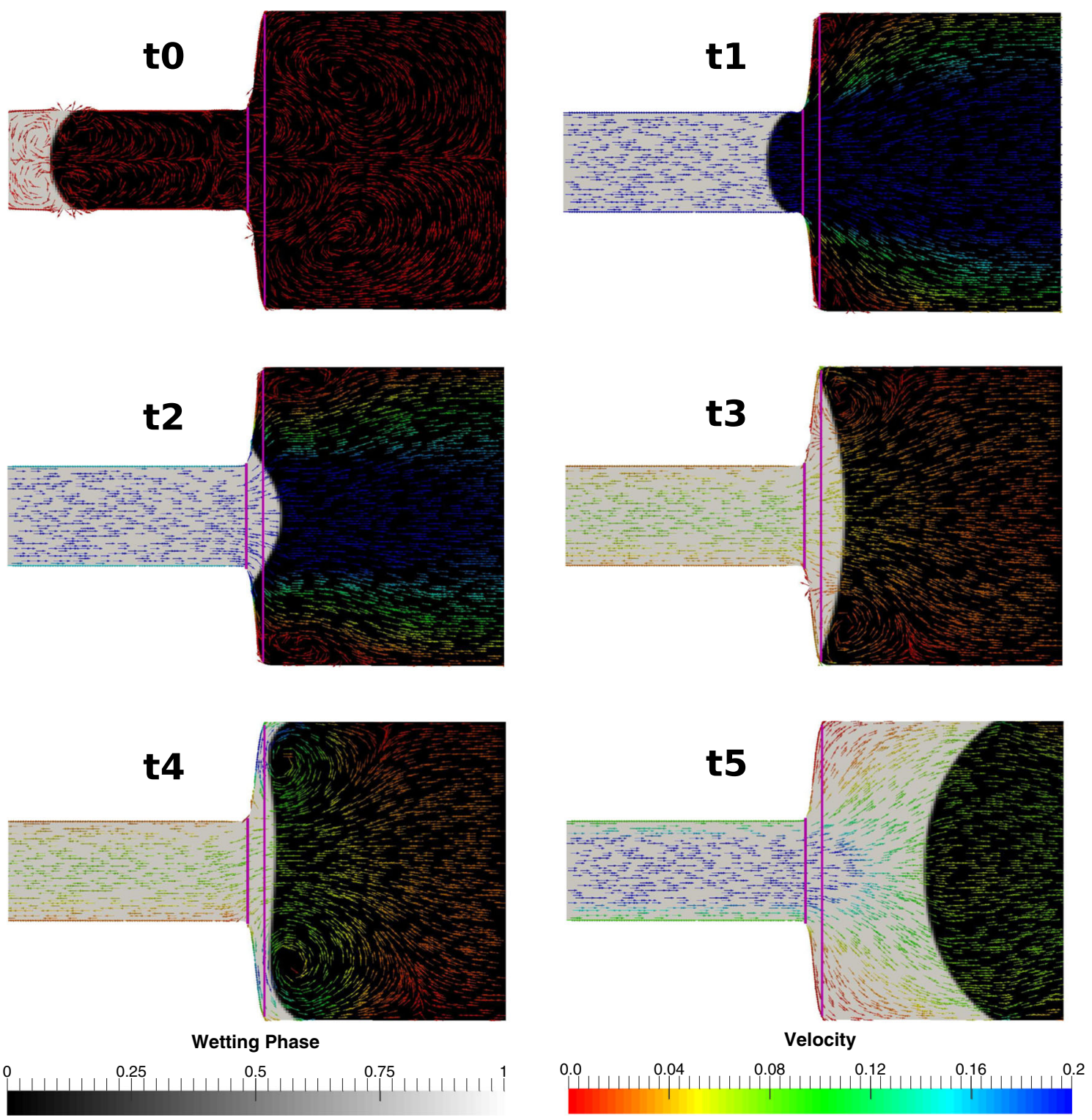

Fig. 11 Spontaneous imbibition for $n=10, \rho_{\alpha}=1000 \mathrm{~kg} / \mathrm{m}^{3} . \theta\left(38^{\circ}\right)>\theta_{c}\left(5.71^{\circ}\right)$. The corresponding time related to the snapshots are $t 0=0.1$ $\mathrm{ms}, t 1=0.2 \mathrm{~ms}, t 2=0.225 \mathrm{~ms}, t 3=0.35 \mathrm{~ms}, t 4=0.4 \mathrm{~ms}$ and $t 5=1 \mathrm{~ms}$

meniscus arrives at the transition zone by gaining velocity gradually over time to reach steady state according to the balance between capillary and viscous forces. At $t 2=$ $0.225 \mathrm{~ms}$, as the meniscus is pushed into the capillary barrier zone due to momentum, the meniscus is subjected to an initial transition in its curvature. At this stage, the capillary and viscous forces act as resisting forces with only inertia driving the flow within the system. At $t 3=$ $0.35 \mathrm{~ms}$, the magnitude of resisting forces grows larger compared to inertia thereby commencing a back flow within the pore geometry. The backward flow tends to create a zone of vortices in the transition zone helping the meniscus temporarily spread over the wall surface. At $t 4=0.4 \mathrm{~ms}$, once the meniscus crosses the capillary barrier zone, the meniscus finds it favourable to spread on the wall and tries to attain an equilibrium configuration according to the contact angle boundary conditions by generating vortices. At $t 5=1 \mathrm{~ms}$, after attaining an equilibrium meniscus configuration, the meniscus gradually accelerates towards steady-state velocity according to the balance between capillary and viscous forces filling the pore body. After entering the capillary barrier zone, if inertia is dominant over resisting forces, stage $t 3$ shown in Fig. 11 is avoided (vortices temporarily spreading the wetting phase on the wall) and the flow profile gradually moves from $t 2$ to $t 4$.

Figure 12 shows the snap-shots of the meniscus position over time for the transition zone shape defined by $n=10$ 

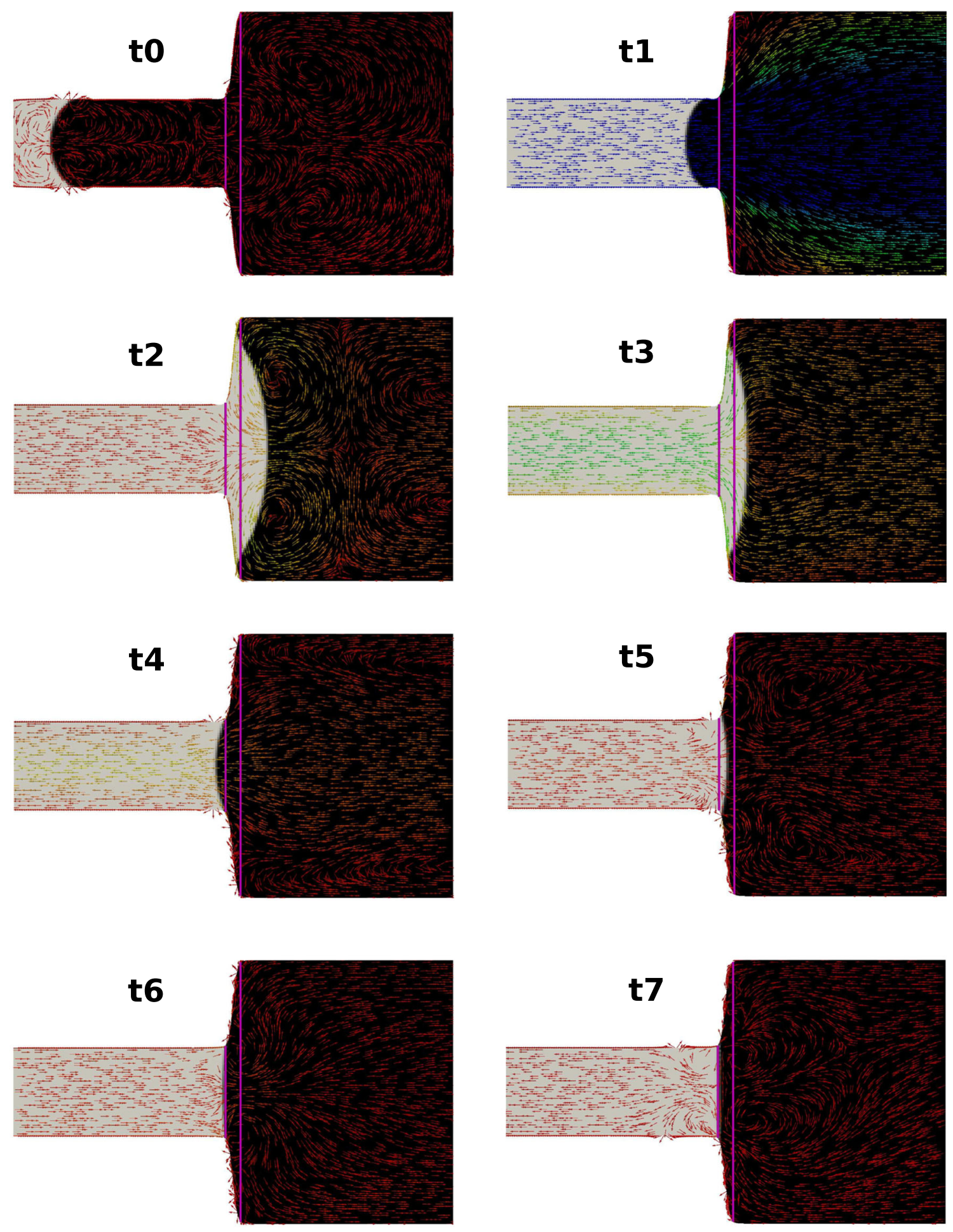

Wetting Phase

\section{Velocity}
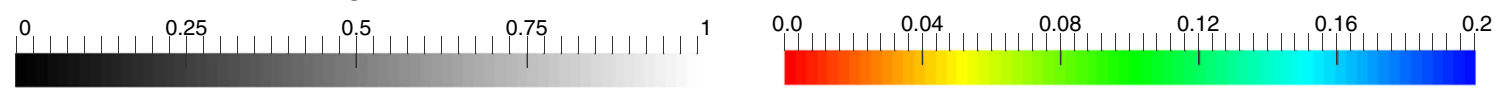

Fig. 12 Spontaneous imbibition for $n=10, \rho_{\alpha}=1000 \mathrm{~kg} / \mathrm{m}^{3} \cdot \theta\left(39^{\circ}\right)>\theta_{c}\left(5.71^{\circ}\right)$. The corresponding time related to the snapshots are $t 0=0.1$ $\mathrm{ms}, t 1=0.2 \mathrm{~ms}, t 2=0.3 \mathrm{~ms}, t 3=0.4 \mathrm{~ms}, t 4=0.525 \mathrm{~ms}, t 5=0.6 \mathrm{~ms}, t 6=0.675 \mathrm{~ms}$ and $t 7=2 \mathrm{~ms}$ 
when the contact angle of the wetting phase is $\theta=39^{\circ}$ which is larger than the critical contact angle, $\theta_{c}=5.71^{\circ}$. For this case, the system marginally falls short of the required energy to push the meniscus over the capillary barrier zone thereby hindering spontaneous imbibition of the pore body. At $t 0=0.1 \mathrm{~ms}$, the meniscus reaches an equilibrium configuration at the end of relaxation stage from where we commence spontaneous imbibition. At $t 1=0.2$ $\mathrm{ms}$, the meniscus arrives at the entrance of the transition zone gradually accelerating to attain steady-state velocity. As the meniscus enters the capillary barrier zone due to inertia, the meniscus is subjected to a change in its curvature. At $t 2=0.3 \mathrm{~ms}$, back flow and vortices are generated as the magnitude of resisting forces (viscous and capillary) grow larger than inertia. The temporary spreading of the meniscus on the wall due to vortices generated during the back flow were not sufficient to push the meniscus over the exit of the capillary barrier zone. At $t 3=0.4$ $\mathrm{ms}$, as capillarity becomes the dominant force within the system, the meniscus is pushed back towards the pore throat. At $t 4=0.525 \mathrm{~ms}$, during the back flow, the momentum would once again push the meniscus over the entrance of the capillary barrier zone into the pore throat. Between $t 4=$ $0.525 \mathrm{~ms}$ to $t 6=0.675 \mathrm{~ms}$, the momentum would repeat the process of pushing the meniscus into the capillary barrier zone and retracting back several times. Over time, these meniscus oscillations around the entrance of the capillary barrier zone dampen. Finally, at $t 7=2 \mathrm{~ms}$, the meniscus settles at the entrance of the capillary barrier zone not invading the pore body.

For inertial dominant flows as seen above, momentum can potentially assist the meniscus overcome the capillary barrier zones even when the contact angle of the wetting phase is greater than the critical contact angle. The inertial dependent contact angle that can overcome the capillary barriers is referred to as the barrier contact angle $\theta_{b}$. From the analysis done for $n=10, \rho_{\alpha}=1000$ $\mathrm{kg} / \mathrm{m}^{3}$, the barrier contact angle lies between $38^{\circ}$ and $39^{\circ}$. Similarly, we determine the barrier contact angle numerically for various inertial systems (considering fluids with different densities) and for different transition zone shapes as shown in Fig. 13. In Fig. 13, the continuous blue line represents the critical contact angle determined by Eq. 16. The numerically determined barrier contact angles for various inertial systems are within $1^{\circ}$ accuracy. For $\rho_{\alpha}=1 \mathrm{~kg} / \mathrm{m}^{3}$, we find a good match between the analytical expression used to determine the critical contact angle and the numerical solution validating the concept put forth in Section 3. If light fluids occupy the system, we see the barrier contact angle move towards the critical contact angle; whereas if the system is occupied by dense fluids, the barrier contact angle is notably larger than the critical contact angle. Note that the numerical analysis shown in

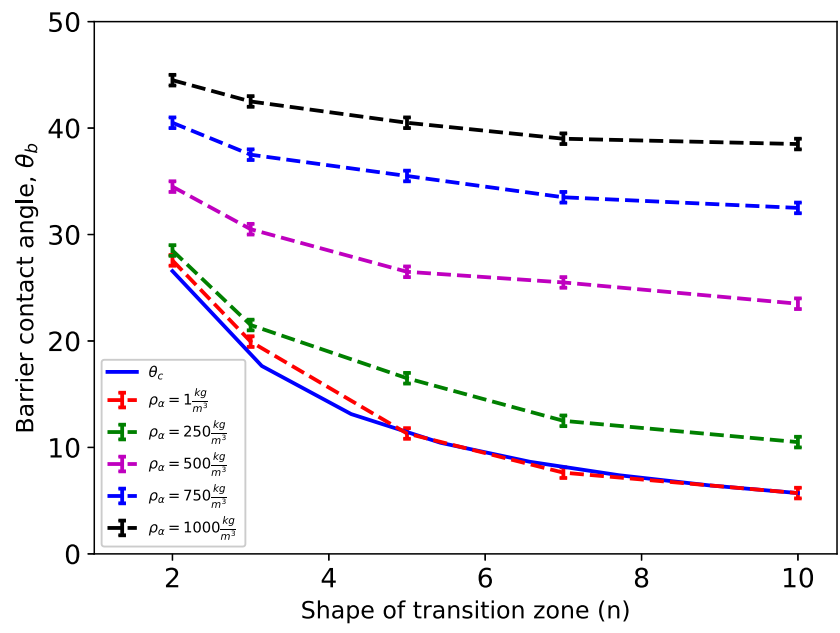

Fig. 13 Barrier contact angle, $\theta_{b}$ for different shapes of the transition zone considering different fluid densities in the system for pore geometry discussed in Fig. 7

Fig. 13 is valid only for the specific pore geometry discussed in Fig. 7. Any change in the pore geometry, boundary conditions or the fluid properties can impact the barrier contact angle.

\subsection{Spontaneous imbibition through a pore doublet}

To investigate the impact of capillary barriers during PBF, we consider a simple pore network (a pore doublet) comprising of two pore bodies having different shapes of transition zone and are of different sizes. Figure 14 illustrates the considered pore doublet along with the spontaneous imbibition boundary conditions of the system that has a single inlet and two outlets connected to both the pore bodies. The wetting phase initially occupies the region enclosed between two solid blue lines. The larger pore body to the right has an aspect ratio of 6 and is twice bigger than the smaller pore body having an aspect ratio of 3 . The scaling factor $s$ used in Eq. 12 for the larger pore body is 2.5. The shape of the transition zone for the larger pore is defined by $n=3$, whereas $n=10$ for the smaller pore. The density of both fluids in the system is $\rho_{\alpha}=500 \mathrm{~kg} / \mathrm{m}^{3}$ (light oil). We compare spontaneous imbibition flow phenomena for the same pore geometry and fluid properties at different contact angles $\left(0^{\circ}\right.$ and $\left.10^{\circ}\right)$. Conventional PNM predict that the smaller pore (left side, $n=10$ ) gets invaded first during imbibition.

Figure 15 shows the snap-shots of the meniscus position during spontaneous imbibition in the considered pore network at different intervals of time when the contact angle of the wetting phase is $10^{\circ}$. The critical contact angle for $n=10$ is $\theta_{c, n=10}=5.71^{\circ}$ and for $n=3$ is $\theta_{c, n=3}=18.4^{\circ}$. For the smaller pore body as the contact angle of the wetting 


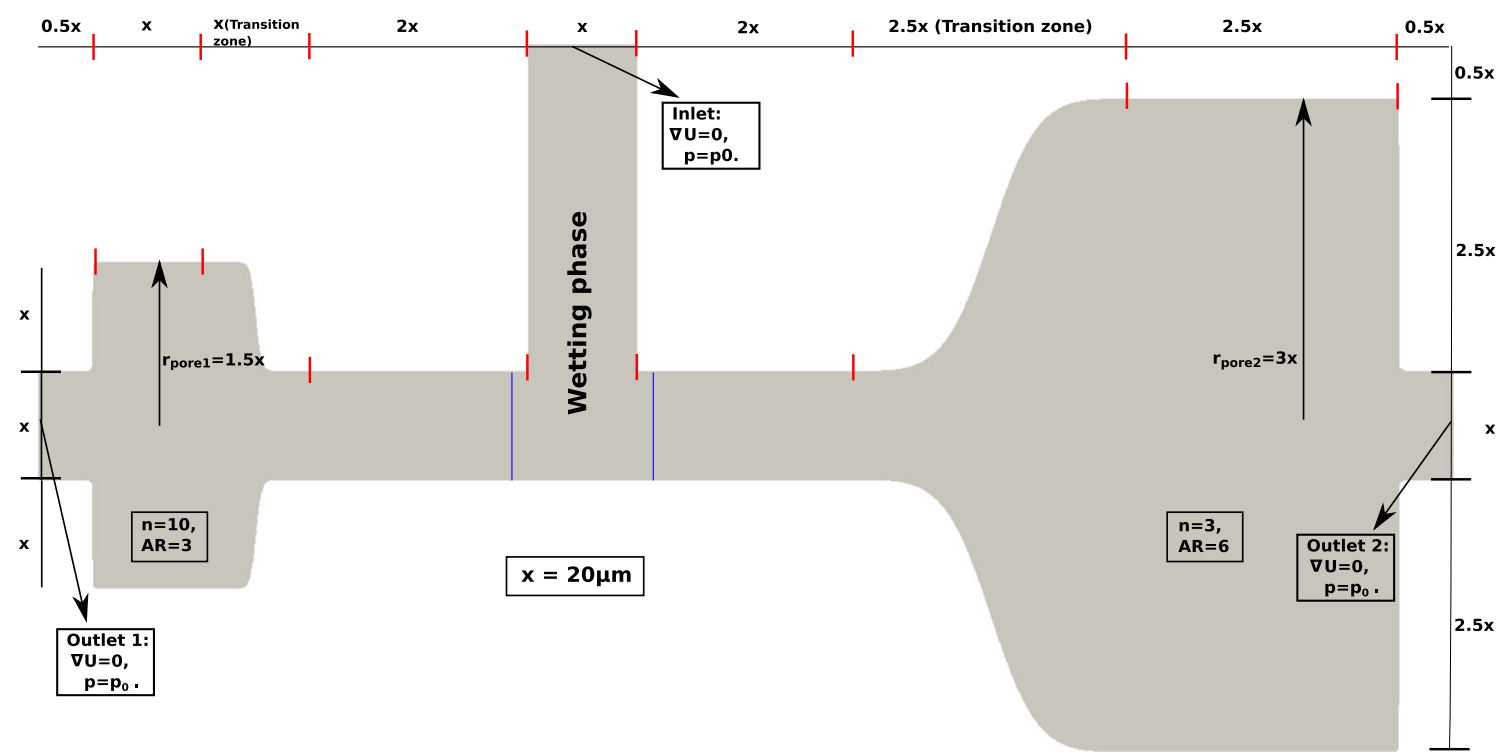

Fig. 14 Illustration of a pore doublet comprising of two pore bodies having different shapes (left: $n=10$, right: $n=3$ ) of transition zone and are of different sizes $\left(r_{p}\right.$,right $=2 \times r_{p}$,left $)$

phase is greater than the critical contact angle $\left(\theta>\theta_{c}\right)$, we expect the presence of a capillary barrier zone. The capillary barrier zone is represented by the region enclosed between pink lines in Fig. 15. The green line in the larger pore body represents the centre of the transition zone for reference. At $t 0=0.1 \mathrm{~ms}$, the meniscus reaches an equilibrium configuration upon relaxation from where we commence spontaneous imbibition. At $t 1=0.4 \mathrm{~ms}$, the capillary forces start to displace both the menisci (left and right) in the horizontal pore throat and the meniscus enters the transition zones of the pore bodies. The meniscus on the left has already reached the entrance of the capillary barrier zone of the smaller pore. On the other hand, for the larger pore, we see the capillary forces still acting (due to the meniscus curvature) and the influx of the wetting phase is directed towards the larger pore. For the larger pore as $\theta<\theta_{c}$, we do not expect any capillary barriers and the meniscus always maintains a curvature (and capillary forces) that keeps displacing the meniscus as seen at $t 2=1 \mathrm{~ms}$ and at $t 3=2.5 \mathrm{~ms}$, where the wetting phase slowly overcomes the centre of the transition zone. At $t 4=6.3 \mathrm{~ms}$, as the meniscus exits the transition zone of the larger pore body and enters the flat horizontal wall surface, according to the contact angle boundary conditions the meniscus tries to attain an equilibrium meniscus configuration by generating vortices. The wetting phase continues to invade the pore body further according to balance between forces within the system. As the meniscus reaches the vertical wall boundary of the larger pore, the meniscus once again adapts according to the contact angle boundary conditions. For the meniscus configurations at $t 5=10.04 \mathrm{~ms}$ and $t 6=12 \mathrm{~ms}$, the capillary forces are greater in the larger pore. As a consequence, the meniscus to the left that has stopped at the entrance of the capillary barrier zone is sucked back slightly towards the pore throat. At $t 5=10.04 \mathrm{~ms}$, as the magnitude of capillary forces in the larger pore body are greater in comparison to the capillary forces at previous time intervals ( $t 1$ to $t 4$ ), the imbibition rate increases thereby eventually filling the larger pore body with the wetting phase as seen at $t 7=20.1 \mathrm{~ms}$. For the smaller pore body, between $t 1$ to $t 7$, the meniscus stays at the entrance of the capillary barrier zone and shows resistance to invade the pore body as there are no capillary (due to the flat meniscus configuration) or external forces acting within the system to overcome the capillary barrier zone. Note that the threshold capillary pressure for the smaller pore body having $\theta>\theta_{c}$ has a negative value similar to the case of $n=5, \theta\left(13^{\circ}\right)>$ $\theta_{c}\left(11.31^{\circ}\right)$ discussed in Section 5.1.1, Fig. 10.

We now consider a case where the contact angle of the wetting phase is $0^{\circ}$ to compare the invasion pattern of the wetting phase when there are no capillary barrier zones during spontaneous imbibition. The numerical setup and fluid properties are the same as discussed for the earlier case (pore doublet with $\theta=10^{\circ}$ ). Figure 16 shows the snap-shots of the meniscus position during spontaneous imbibition in the considered pore doublet at different intervals of time. For the considered contact angle $\theta=0^{\circ}$, there are no capillary barrier zones. The pink lines represent the centre of the transition zone for reference. At $t 1=1 \mathrm{~ms}$, after relaxing the meniscus and commencing spontaneous imbibition, the wetting phase invades the horizontal pore throats and commences to enter the transition zone of both pores. At $t 2=2.5 \mathrm{~ms}$, the meniscus to the left in the smaller pore body crosses the transition zone entering a favourable 

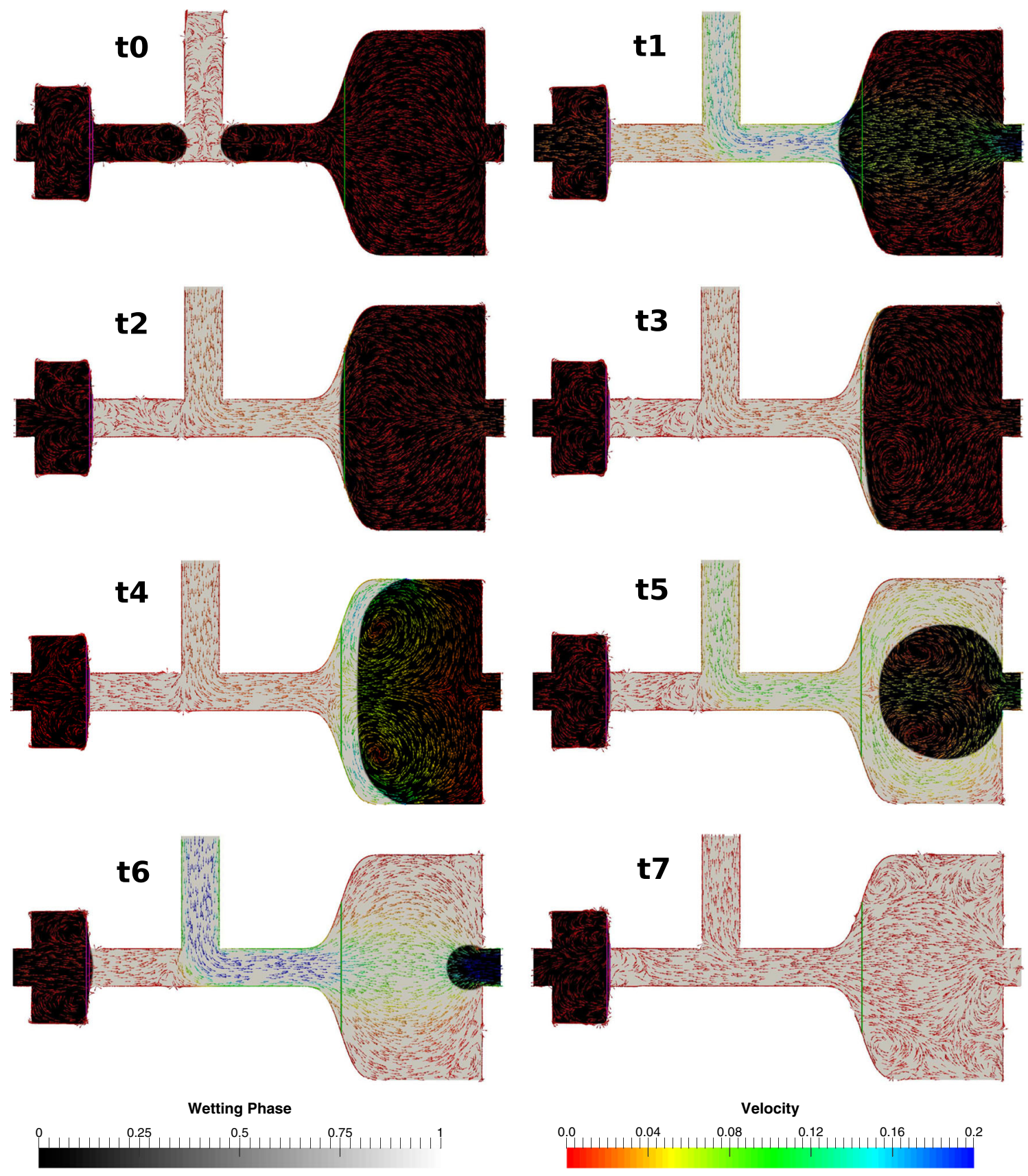

Fig. 15 Spontaneous imbibition in a pore doublet with contact angle, $\theta=10^{\circ}$. Unlike conventional PNM that predict invading smaller pore initially, the impact of wall shape and contact angle show preference to invade the larger pore body and not imbibe the smaller pore at all due

to the capillary barrier effect. Corresponding time related to the snapshots are $t 0=0.1 \mathrm{~ms}, t 1=0.4 \mathrm{~ms}, t 2=1 \mathrm{~ms}, t 3=2.5 \mathrm{~ms}, t 4=6.3$ $\mathrm{ms}, t 5=10.04 \mathrm{~ms}, t 6=12 \mathrm{~ms}$ and $t 7=20.1 \mathrm{~ms}$

wall surface to spread; whereas the meniscus to the right is close to exit the transition zone of the larger pore body. Note that the meniscus curvature on the right is less compared to the meniscus curvature on the left resulting in relatively weaker capillary forces on the right. Between $t 3=3.5$ $\mathrm{ms}$ and $t 4=3.7 \mathrm{~ms}$, as a consequence of the competition between capillary forces at both ends, the left pore body gets imbibed and sucks back the meniscus on the right.

Later, at $t 5=5 \mathrm{~ms}$ and $t 6=6 \mathrm{~ms}$, the meniscus on the right tries to attain equilibrium and starts filling up the larger pore body. As seen in the earlier case, the meniscus configures itself according to the contact angle conditions upon encountering the vertical wall surface at seen at $t 7=9$ ms. Finally at $t 8=10.04 \mathrm{~ms}$, due to stronger capillary forces at $t 7$, the wetting phase quickly imbibes the larger pore body. 

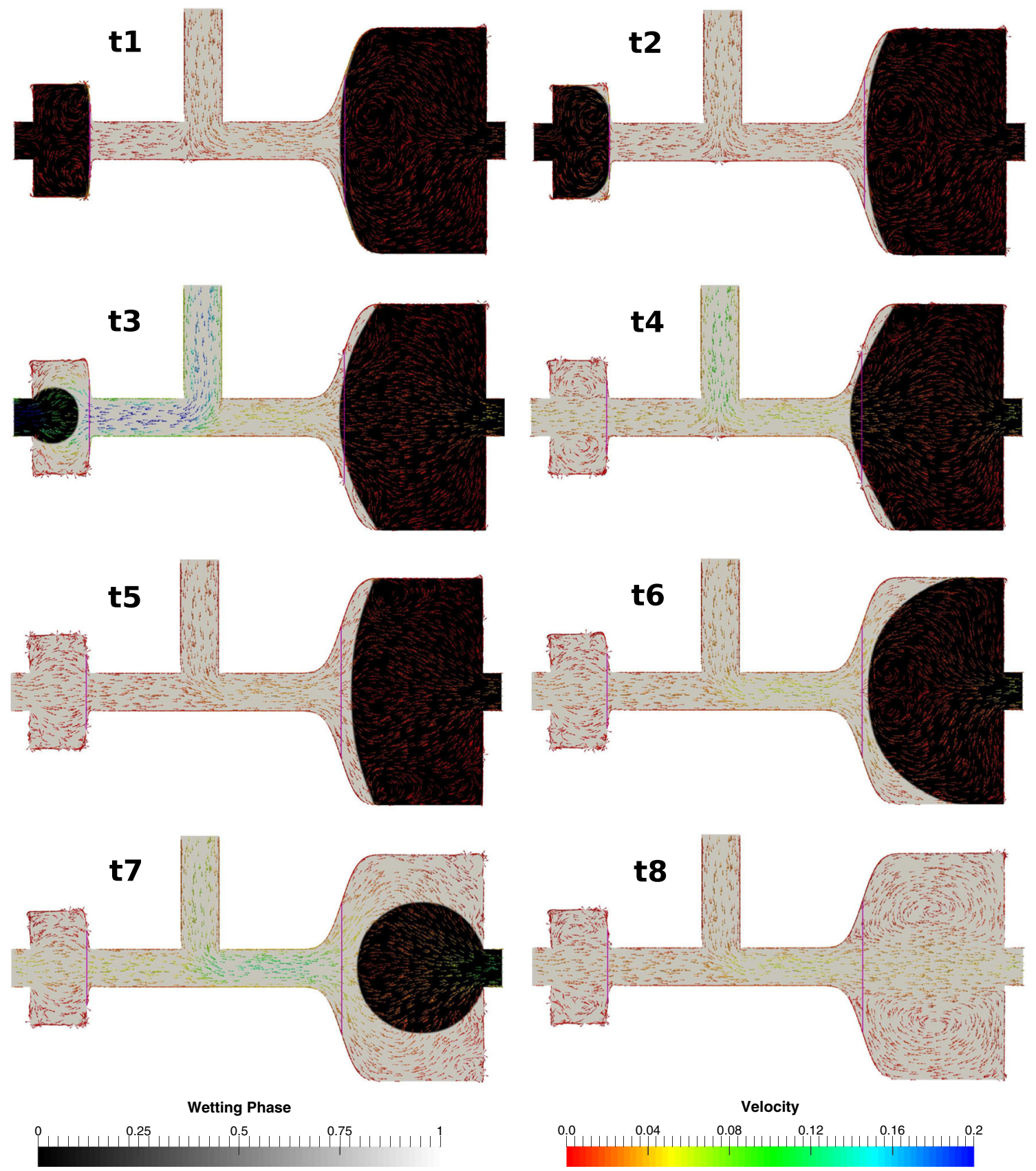

Fig. 16 Spontaneous imbibition in a pore doublet with contact angle, $\theta=0^{\circ} . \theta=0^{\circ}$ results in no capillary barriers. Corresponding time related to the snap-shots are $t 1=1 \mathrm{~ms}, t 2=2.5 \mathrm{~ms}, t 3=3.5 \mathrm{~ms}, t 4=3.7 \mathrm{~ms}, t 5=5 \mathrm{~ms}, t 6=6 \mathrm{~ms}, t 7=9 \mathrm{~ms}$ and $t 8=10.04 \mathrm{~ms}$

This case study of a pore doublet clearly demonstrates that imbibition is strongly dependent on the shape of the pore geometry along with the contact angle of the wetting phase and contradicts the conventional theory that smaller pores are always preferred during imbibition. This can have major implications in choosing the invasion paths and potentially impact determining the relative permeabilities of fluids.

Based on the observations from the pore singlet (weak inertial systems, Section 5.1.1) and pore doublet case studies, when there are no capillary barriers $\left(\theta<\theta_{c}\right)$, recovery of the non-wetting phase is $100 \%$. On the other hand, when a capillary barrier exists $\left(\theta>\theta_{c}\right)$, the nonwetting phase is not fully recovered. For example, in the pore doublet case when a capillary barrier exists for the smaller pore body, the non-wetting phase recovery drops to $86.7 \%$. Extending our observation to a larger macroscopic system, we speculate that the presence of capillary barriers will have an impact on the recovery of the non-wetting phase during spontaneous imbibition flows. 


\section{Conclusion}

This study exclusively focuses on the event of pore body filling and we revisit the basic principles governing the invasion of a pore body during spontaneous imbibition. Conventional pore filling rules take into account only the sizes of pores and throats and additionally comprise of stochastic parameters that introduce uncertainties to model imbibition. In our study, we find that the geometric characteristics of the pore space, contact angle and the fluid properties play an important role during imbibition. The relationship between meniscus dynamics and factors mentioned above are quantitatively analysed. For this study, we consider a simple 2D synthetic pore geometry with varying shapes of the transition zone (pore space connecting a pore throat and a pore body) and use direct numerical simulations to investigate water flooding. The main findings of this work are the following: (1) capillary barriers are seen to play a significant role in choosing the invasion paths of the wetting phase during spontaneous imbibition. The presence of capillary barrier zones are linked to the shape of transition zone and contact angle of the fluid, (2) meniscus oscillations that were previously investigated during forced and spontaneous imbibition on pores having sharp transition zone shapes [8, $25]$ are also seen to occur for smooth transition zones. We unravel the physics behind these transitions in the meniscus curvature during imbibition that are linked to the capillary barriers. We also notice that for pores having capillary barrier zones the threshold capillary pressure is a negative value and (3) we show how the invading phase can overcome the capillary barriers during spontaneous imbibition due to inertia.

For the investigated pore geometries, we demonstrate the existence of a critical contact angle $\theta_{c}$ that varies according to the slope of the wall boundary in the transition zone. We provide an analytical expression to determine the critical contact angle for the investigated cases. The relation between contact angle of the wetting phase $\theta$ and the critical contact angle $\theta_{c}$ decides if the wetting phase can imbibe a pore body. Imbibition always happens when $\theta<\theta_{c}$. For $\theta \geq \theta_{c}$, capillary barrier zones occur. Once the meniscus enters capillary barrier zones, the capillary forces try to resist imbibition. It is important to investigate the parameters that result in the occurrence of capillary barrier zones as their presence can potentially hinder imbibition and influence the invasion paths. We provide a semianalytic expression to determine the position of the capillary barrier zones for the investigated case. During spontaneous imbibition, inertia can assist the wetting phase to overcome the capillary barrier zones at a barrier contact angle $\theta_{b}$ that we determine numerically for the geometries we consider. For inertia-dominated systems (system occupied by heavy fluids), $\theta_{b}$ is notably larger than than $\theta_{c}$; whereas for systems occupied by light fluids, $\theta_{b}$ is slightly larger than $\theta_{c}$. We consider the impact of this study on a simple pore network comprising of two pores having different sizes and shapes. Unlike conventional pore network models which predict invading a smaller pore body, we rather observe the larger pore body getting invaded first and the smaller pore body never gets invaded by the wetting phase due to the capillary barrier effect.

This study can be an important step towards a deeper understanding of the spontaneous imbibition event in pore geometries. The analysis of this study can be used to improve the existing pore filling rules that incorporates flow physics and can lead to improved predictive capabilities of pore network model simulations.

Acknowledgements The authors would like to thank TOTAL management for the authorisation to publish this work.

Open Access This article is distributed under the terms of the Creative Commons Attribution 4.0 International License (http:// creativecommons.org/licenses/by/4.0/), which permits unrestricted use, distribution, and reproduction in any medium, provided you give appropriate credit to the original author(s) and the source, provide a link to the Creative Commons license, and indicate if changes were made.

\section{References}

1. Afkhami, S., Zaleski, S., Bussmann, M.: A mesh-dependent model for applying dynamic contact angles to vof simulations. J. Comput. Phys. 228(15), 5370-5389 (2009)

2. Batchelor, G.K.: An introduction to fluid dynamics. Cambridge University Press, Cambridge (2000)

3. Berthier, J., Brakke, K.A.: The physics of microdroplets. Wiley, New York (2012)

4. Blunt, M.J.: Multiphase flow in permeable media: A pore-scale perspective. Cambridge University Press, Cambridge (2017)

5. Brackbill, J., Kothe, D.B., Zemach, C.: A continuum method for modeling surface tension. J. Comput. Phys. 100(2), 335-354 (1992)

6. Denner, F., Wachem, B.G.: Numerical time-step restrictions as a result of capillary waves. J. Comput. Phys. 285, 24-40 (2015)

7. Dong, H., Blunt, M.J.: Pore-network extraction from microcomputerized-tomography images. Phys. Rev. E 80(3), 036307 (2009)

8. Ferrari, A., Lunati, I.: Inertial effects during irreversible meniscus reconfiguration in angular pores. Adv. Water Resour. 74, 1-13 (2014)

9. Graue, A., Viksund, B.G., Eilertsen, T., Moe, R.: Systematic wettability alteration by aging sandstone and carbonate rock in crude oil. J. Pet. Sci. Eng. 24(2-4), 85-97 (1999)

10. Hirt, C.W., Nichols, B.D.: Volume of fluid (vof) method for the dynamics of free boundaries. J. Comput. Phys. 39(1), 201-225 (1981)

11. Huh, C., Scriven, L.: Hydrodynamic model of steady movement of a solid/liquid/fluid contact line. J. Colloid Interface Sci. 35(1), 85-101 (1971)

12. Issa, R.I.: Solution of the implicitly discretised fluid flow equations by operator-splitting. J. Comput. Phys. 62(1), 40-65 (1986) 
13. Lenormand, R., Zarcone, C., Sarr, A.: Mechanisms of the displacement of one fluid by another in a network of capillary ducts. J. Fluid Mech. 135, 337-353 (1983)

14. Lenormand, R., Zarcone, C., et al.: Role of Roughness and Edges during Imbibition in Square Capillaries. In: SPE Annual Technical Conference and Exhibition. Society of Petroleum Engineers (1984)

15. Mathematica, W.: Wolfram Research. Illinois, Inc., Champaign (2018)

16. Méheust, Y., Løvoll, G., Måløy, K.J., Schmittbuhl, J.: Interface scaling in a two-dimensional porous medium under combined viscous, gravity, and capillary effects. Phys. Rev. E 66(5), 051603 (2002)

17. Navier, C.: Mémoire sur les lois du mouvement des fluides. Mem. Acad. Sci. Inst. Fr 6(1823), 389-416 (1823)

18. Pavuluri, S., Maes, J., Doster, F.: Spontaneous imbibition in a microchannel: analytical solution and assessment of volume of fluid formulations. Microfluid. Nanofluid. 22(8), 90 (2018)

19. Raeini, A.Q., Blunt, M.J., Bijeljic, B.: Modelling two-phase flow in porous media at the pore scale using the volume-of-fluid method. J. Comput. Phys. 231(17), 5653-5668 (2012)

20. Raeini, A.Q., Blunt, M.J., Bijeljic, B.: Direct simulations of twophase flow on micro-ct images of porous media and upscaling of pore-scale forces. Adv. Water Resour. 74, 116-126 (2014)

21. Rusche, H.: Computational fluid dynamics of dispersed two-phase flows at high phase fractions. Ph.D. thesis, Imperial College London (University of London) (2003)
22. Singh, K., Bijeljic, B., Blunt, M.J.: Imaging of oil layers, curvature and contact angle in a mixed-wet and a water-wet carbonate rock. Water Resour. Res. 52(3), 1716-1728 (2016)

23. Singh, K., Menke, H., Andrew, M., Lin, Q., Rau, C., Blunt, M.J., Bijeljic, B.: Dynamics of snap-off and pore-filling events during two-phase fluid flow in permeable media. Sci. Rep. 7(1), 5192 (2017)

24. Sinha, P.K., Wang, C.Y.: Pore-network modeling of liquid water transport in gas diffusion layer of a polymer electrolyte fuel cell. Electrochim. Acta 52(28), 7936-7945 (2007)

25. Zacharoudiou, I., Chapman, E.M., Boek, E.S., Crawshaw, J.P.: Pore-filling events in single junction micro-models with corresponding lattice boltzmann simulations. Journal of Fluid Mechanics (2017)

26. Zhao, B., MacMinn, C.W., Juanes, R.: Wettability control on multiphase flow in patterned microfluidics. Proc. Natl. Acad. Sci. 113(37), 10251-10256 (2016)

27. Zhou, X., Morrow, N.R., Ma, S., et al.: Interrelationship of wettability, initial water saturation, aging time, and oil recovery by spontaneous imbibition and waterflooding. SPE J. 5(02), 199-207 (2000)

Publisher's note Springer Nature remains neutral with regard to jurisdictional claims in published maps and institutional affiliations.

\section{Affiliations}

\section{Saideep Pavuluri ${ }^{1}$ (D) $\cdot$ Julien Maes $^{1} \cdot$ Jianhui Yang $^{2} \cdot$ Mohamed Regaieg $^{2} \cdot$ Arthur Moncorgé $^{2} \cdot$ Florian Doster $^{1}$}

1 Institute of Petroleum Engineering, Heriot-Watt University, Edinburgh, EH14 4AS, UK

2 Geoscience Research Centre, Total E, P UK, Westhill, AB32 6JZ, UK 